\title{
Stability of local quantum dissipative systems
}

\author{
Toby S. Cubitt*1,2, Angelo Lucia ${ }^{\dagger 1}$, Spyridon Michalakis ${ }^{\ddagger 3}$, and David Perez-Garcia ${ }^{\S 1}$ \\ ${ }^{1}$ Departamento de Análisis Matemático, Universidad Complutense de Madrid, \\ 28040 Madrid, Spain \\ ${ }^{2}$ DAMTP, University of Cambridge, Centre for Mathematical Sciences, \\ Cambridge CB3 0WA, United Kingdom \\ ${ }^{3}$ Institute for Quantum Information and Matter, Caltech, Pasadena, CA 91125, USA
}

\begin{abstract}
Open quantum systems weakly coupled to the environment are modeled by completely positive, trace preserving semigroups of linear maps. The generators of such evolutions are called Lindbladians. In the setting of quantum many-body systems on a lattice it is natural to consider Lindbladians that decompose into a sum of local interactions with decreasing strength with respect to the size of their support. For both practical and theoretical reasons, it is crucial to estimate the impact that perturbations in the generating Lindbladian, arising as noise or errors, can have on the evolution. These local perturbations are potentially unbounded, but constrained to respect the underlying lattice structure. We show that even for polynomially decaying errors in the Lindbladian, local observables and correlation functions are stable if the unperturbed Lindbladian has a unique fixed point and a mixing time which scales logarithmically with the system size. The proof relies on Lieb-Robinson bounds, which describe a finite group velocity for propagation of information in local systems. As a main example, we prove that classical Glauber dynamics is stable under local perturbations, including perturbations in the transition rates which may not preserve detailed balance.
\end{abstract}

\section{Contents}

1 Background and previous work 2

2 Stability of open quantum systems

3 Setup and notation 4

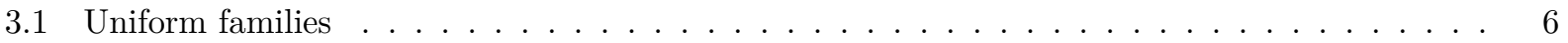

4 Main result 8

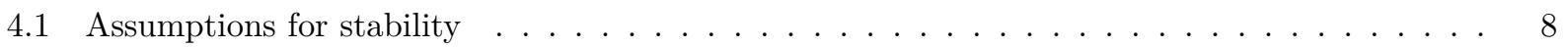

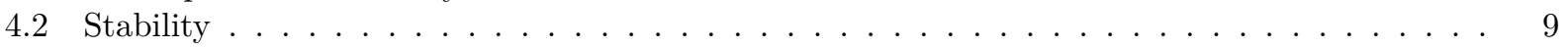

4.3 Local observables vs. global observables . . . . . . . . . . . . . . . . . . 10

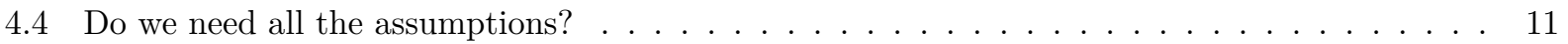

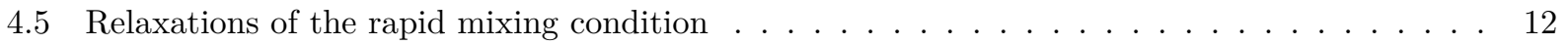

*tsc25@cam.ac.uk

†anlucia@ucm.es

†spiros@caltech.edu

§dperezga@ucm.es 
5 Toolbox for the proof 12

5.1 Lieb-Robinson bounds for Lindbladian evolution . . . . . . . . . . . . . . . . . . . 13

5.2 Local rapid mixing . . . . . . . . . . . . . . . . . . . . . . . . . . 17

6 Proof of main result 17

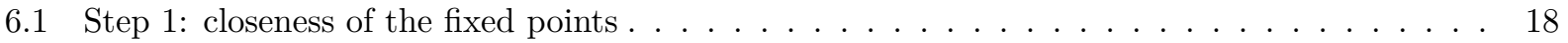

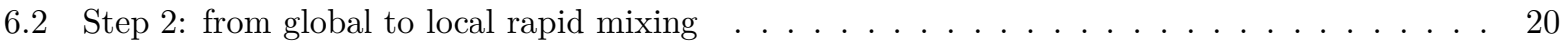

6.3 Step 3: from local rapid mixing to stability $\ldots \ldots \ldots \ldots \ldots \ldots \ldots \ldots \ldots \ldots$

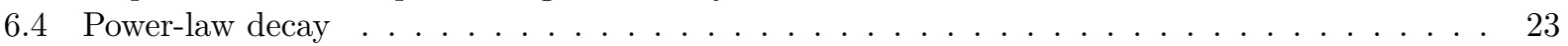

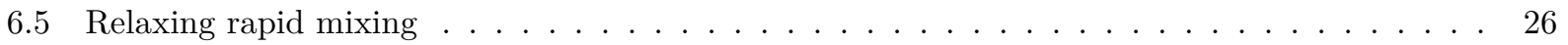

7 Glauber dynamics 27

7.1 Quantum embedding of Glauber dynamics . . . . . . . . . . . . . . . . . 27

7.2 Stability of Glauber dynamics . . . . . . . . . . . . . . . . . . . 31

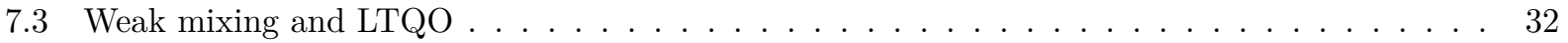

8 Conclusions and open questions

\begin{tabular}{ll} 
Appendices & 33 \\
\hline
\end{tabular}

Appendix A The non-stable example 33

\section{Background and previous work}

The physical properties of a closed many-body quantum system are encoded in its Hamiltonian. Theoretical models of such systems typically assume some form of local structure, whereby the Hamiltonian decomposes into a sum over interactions between subsets of nearby particles. Similarly, the behavior of an open manybody quantum system is encoded in its Liouvillian. Again, this is typically assumed to have a local structure, decomposing into a sum over local Liouvillians acting on subsets of nearby particles.

Crucial to justifying such theoretical models is the question of whether their physical properties are stable under small perturbations to the local interactions. If the physical properties of a many-body Hamiltonian or Liouvillian depend sensitively on the precise mathematical form of those local terms, then it is difficult to conclude anything about physical systems, whose interactions will always deviate somewhat from theory.

Quantum information theory has motivated another perspective on many-body Hamiltonians. Rather than studying models of naturally occurring systems, it studies how many-body systems can be engineered to produce desirable behavior, such as long-term storage of information in quantum memories $12,19,20$, [48, processing of quantum information for quantum computing [11, 13, 14, 34, 51], or simulation of other quantum systems which are computationally intractable by classical means $[3,7,8,28,30$. Again, stability of these systems under local perturbations is crucial, otherwise even tiny imperfections may destroy the desired properties. Stability in this context has been studied for self-correcting topological quantum memories, where one in addition requires robustness against local sources of dissipative noise, and the relevant quantity is the minimum time needed to introduce logical errors in the system. It has been known since [1, 12] that a self-correcting quantum memory with local interactions is possible in four spatial dimensions. With the breakthrough of the Haah code [19], it seems it may be possible to engineer such self-correcting quantum memories in three dimensions.

Recently, and partially motivated by the dissipative nature of noise, this "engineering" approach has been extended to open quantum systems and many-body Liouvillians. First theoretical [38, 61], and then experimental 5. 39] work has shown that creating many-body quantum states as fixed points of engineered, dissipative Markovian evolutions can be more robust against undesirable errors and maintain coherence of quantum information for longer times. Intuitively, there is an inherent robustness in such models: the target state is independent of the initial state. If the dissipation is engineered perfectly, the system will always be driven back towards the desired state. This idea can be used to engineer dissipative systems 
both for storing quantum information [38, 61] and for carrying out computation via dissipative dynamics 61]. However, it does not guarantee stability against errors in the engineered Liouvillian itself. Once again, stability against local perturbations - this time for many-body Liouvillians rather than Hamiltonians - is of crucial importance.

In the case of closed systems governed by Hamiltonians, recent breakthroughs have given rigorous mathematical justification to our intuition that the physical properties of many-body Hamiltonians are stable. Starting with [10, 35], it culminated in the work of [49] which showed that, under a set of mathematically well-defined and physically reasonable conditions, gapped many-body Hamiltonians are stable under perturbations to the local interactions 11 More precisely, in the presence of frustration-freeness, local topological quantum order, and local gap, the spectral gap of a Hamiltonian with local (or quasi-local) interactions is stable against small (quasi-) local perturbations (see [49] for a formal definition of these conditions). The bound on the amount of imperfection tolerated by the system depends on the decay of the local gaps, the decay of the local topological order, and the strength (and decay rate) of the interactions. Furthermore, except for frustration freeness which is a technical condition required in the proof, these conditions are in a sense tight. There exist simple counterexamples to stability if any one of the conditions is lifted.

\section{Stability of open quantum systems}

In this work, we study stability of many-body Liouvillians. We consider dynamics generated by rapidly decaying interactions, where the notion of rapid decay is made precise in section 3. Moreover, in order to have a well-defined notion of scaling with system size, we restrict to Liouvillians whose local terms depend only on the subsystem on which they act, and thus are not redefined as we consider larger systems. We call such families of Liouvillians uniform.

Our main result shows that, under the above assumptions on the structure of the Liouvillian, logarithmic mixing time implies the desired stability in the dissipative setting.

However, although the result is analogous to [49], the proof and even the definition of stability in the case of Liouvillians necessarily differ substantially from the Hamiltonian case. For Hamiltonians, the relevant issue is stability of the spectral gap. Via the quasi-adiabatic technique [22, 24], this in turn implies a smooth transition between the initial and perturbed ground states, showing that both are within the same phase. Note that the existence of a smooth transition (no closing of the spectral gap in the thermodynamic limit) does not imply that both ground states are close in norm, as the simple example $H=\sum_{i=1}^{N}|0\rangle\left\langle\left. 0\right|_{i}\right.$ vs. $H(\varepsilon)=\sum_{i=1}^{N}(|0\rangle+\varepsilon|1\rangle)(\langle 0|+\varepsilon\langle 1|)_{i} /\left(1+\varepsilon^{2}\right)$ shows ${ }^{2}$ It does however imply a well-behaved perturbation in the expectation value of local observables - such as order parameters - and correlation functions, which in most experimental situations are the only measurable quantities.

For Liouvillians, we are interested in a definition of stability more related to the evolution itself, which accounts at the same time for both the speed of convergence and the properties of the fixed point. Here, we consider the strongest definition of stability: we want our systems (initial and perturbed) to evolve similarly for all times and all possible initial states. Thus, not only should the speed of convergence to the fixed points be similar, the fixed points themselves should be close and so should the approach to the fixed points.

This definition is significantly stronger than stability of the spectral gap alone $3^{3}$ and is more directly relevant to the applications discussed above. As in the Hamiltonian case, the analogous simple example shows that one cannot expect to attain such stability if we consider global measurements on the system. Therefore, in analogy with the Hamiltonian case, restrict our attention to local observables and few-body correlation functions. Since there are technical subtleties involved in extending this stronger definition of

\footnotetext{
${ }^{1}$ Note that, in stark contrast to traditional perturbation theory, the perturbations considered here simultaneously change all the local interactions by a small amount. The strength of the total perturbation therefore scales with system size and standard perturbation theory does not apply. It is the structure of local ground states of the Hamiltonian that ensures stability.

${ }^{2}$ Note that each Hamiltonian is the sum of non-interacting projections for any $\varepsilon \in \mathbb{R}$. In particular, for each $\varepsilon$, there is a unitary $U(\varepsilon)$ acting on a single site, such that $H(\varepsilon)=U(\varepsilon)^{\otimes N} H U^{\dagger}(\varepsilon)^{\otimes N}$.

${ }^{3}$ Due to the recent work in [58], it is not clear whether the spectral gap in Liouvillians is the relevant quantity for convergence questions.
} 
stability to dynamics with multiple fixed points, we defer consideration of multiple fixed points to a future paper, and restrict our attention here to dissipative dynamics with unique fixed points. It is important to note, however, that we do not make any assumption on the form of the unique fixed point. In particular, we do not assume that it is full-rank (primitivity); our results apply equally well to Liouvillians with pure fixed points. (Pure-state fixed points are particularly relevant to quantum information applications, such as dissipative state engineering and computation.)

A key technical ingredient in the stability proof for Hamiltonians is the quasi-adiabatic evolution technique 22, 24], which directly uses the fact that Hamiltonian evolution is reversible. This is of course no longer true for Liouvillians, so we must use a different proof approach. We make use of the fact that evolution under a Liouvillian converges to a steady-state, together with dissipative generalizations [50] of the Lieb-Robinson bounds that are the other crucial ingredient in 49 .

Among systems which satisfy our assumptions, one finds classical Glauber dynamics 47]. This immediately shows that Glauber dynamics is stable against errors. To the best of our knowledge, this is new even to the classical literature (related results, but with different assumptions, were given in 27]). Given the importance of Glauber dynamics to sampling from the thermal distributions of classical spin systems 41 , 47, we expect our results to have applications also to classical statistical mechanics.

The paper is structured as follows: After setting up notation and basic definitions in the next section, we state our main stability result in section 4 and discuss the assumptions it requires. In section 5 we prove various technical results used in the main proof, which is given in section 6. We apply these results in section 7 to the important example of classical Glauber dynamics, before concluding with a discussion of the results and related open questions in section 8 .

\section{Setup and notation}

We will consider a cubic lattice $\ell^{4} \Gamma=\mathbb{Z}^{D}$. The ball centered at $x \in \Lambda$ of radius $r$ will be denoted by $b_{x}(r)$. At each site $x$ of the lattice we will associate one elementary quantum system with a finite dimensional Hilbert space $\mathcal{H}_{x}$. We will use the Dirac notation for vectors: $|\phi\rangle$ will denote a vector in $\mathcal{H}_{x},\langle\phi|$ its adjoint, and $\{|n\rangle\}_{n=0}^{\operatorname{dim} \mathcal{H}_{x}}$ the canonical basis for $\mathcal{H}_{x}$. Scalar product in $\mathcal{H}_{x}$ will be denoted by $\langle\phi \mid \psi\rangle$, and rank-one linear maps by $|\phi\rangle\langle\psi|$. For each finite subset $\Lambda \subseteq \Gamma$, the associated Hilbert space is given by

$$
\mathcal{H}_{\Lambda}=\bigotimes_{x \in \Lambda} \mathcal{H}_{x}
$$

and the algebra of observables supported on $\Lambda$ is defined by

$$
\mathcal{A}_{\Lambda}=\bigotimes_{x \in \Lambda} \mathcal{B}\left(\mathcal{H}_{x}\right)
$$

If $\Lambda_{1} \subset \Lambda_{2}$, there is a natural inclusion of $\mathcal{A}_{\Lambda_{1}}$ in $\mathcal{A}_{\Lambda_{2}}$ by identifying it with $\mathcal{A}_{\Lambda_{1}} \otimes \mathbb{1}$. The support of an observable $O \in \mathcal{A}_{\Lambda}$ is the minimal set $\Lambda^{\prime}$ such that $O=O^{\prime} \otimes \mathbb{1}$, for some $O^{\prime} \in \mathcal{A}_{\Lambda^{\prime}}$, and will be denoted by $\operatorname{supp} O$. We will denote by $\|\cdot\|_{p}$ the Schatten $p$-norm over $\mathcal{A}_{\Lambda}$. Where there is no risk of ambiguity, $\|\cdot\|$ will denote the usual operator norm (i.e. the Schatten $\infty$-norm).

A linear map $\mathcal{T}: \mathcal{A}_{\Lambda} \rightarrow \mathcal{A}_{\Lambda}$ will be called a superoperator to distinguish it from operators acting on states. The support of a superoperator $\mathcal{T}$ is the minimal set $\Lambda^{\prime} \subseteq \Lambda$ such that $\mathcal{T}=\mathcal{T}^{\prime} \otimes \mathbb{1}$, where $\mathcal{T}^{\prime} \in \mathcal{B}\left(\mathcal{A}_{\Lambda^{\prime}}\right)$. A superoperator is said to be Hermiticity preserving if it maps Hermitian operators to Hermitian operators. It is said to be positive if it maps positive operators (i.e. operators of the form $O^{*} O$ ) to positive operators. $\mathcal{T}$ is called completely positive if $\mathcal{T} \otimes \mathbb{1}: \mathcal{A}_{\Lambda} \otimes M_{n} \rightarrow \mathcal{A}_{\Lambda} \otimes M_{n}$ is positive for all $n \geqslant 1$. Finally, we say that $\mathcal{T}$ is trace preserving if $\operatorname{tr} \mathcal{T}(\rho)=\operatorname{tr} \rho$ for all $\rho \in A_{\Lambda}$. For a general review on superoperators, see 63.

The dynamics of the system is generated by a superoperator $\mathcal{L}$, which plays a similar role to the Hamiltonian in the non-dissipative case. The evolution will be given by the one parameter semigroup $T_{t}=e^{t \mathcal{L}}$.

\footnotetext{
${ }^{4}$ We restrict to cubic lattices for the sake of exposition. The results can be extended to more general settings, replacing the lattice $\mathbb{Z}^{D}$ with a graph with polynomial growth.
} 
The natural assumptions to make about $T_{t}$ are that it is a continuous semigroup of completely positive and trace preserving maps (CPTP, sometimes also called quantum channels). Such maps are always contractive, meaning that $\left\|T_{t}\right\|_{1 \rightarrow 1, c b} \leqslant 1$, where the completely-bounded norm is defined as:

$$
\|T\|_{1 \rightarrow 1, c b}=\sup _{n}\left\|T \otimes \mathbb{1}_{n}\right\|_{1 \rightarrow 1}=\sup _{n} \sup _{\substack{X \in \mathcal{A}_{\Lambda} \otimes M_{n} \\ X \neq 0}} \frac{\left\|T \otimes \mathbb{1}_{n}(X)\right\|_{1}}{\|X\|_{1}} .
$$

We will also be interested in the $\|\cdot\|_{\infty \rightarrow \infty, c b}$ completely-bounded norm of superoperators, which is defined as follows:

$$
\|T\|_{\infty \rightarrow \infty, c b}=\sup _{n}\left\|T \otimes \mathbb{1}_{n}\right\|_{\infty \rightarrow \infty}=\sup _{n} \sup _{\substack{X \in \mathcal{A}_{\Lambda} \otimes M_{n} \\ X \neq 0}} \frac{\left\|T \otimes \mathbb{1}_{n}(X)\right\|_{\infty}}{\|X\|_{\infty}} .
$$

The relationship between $\|\cdot\|_{1 \rightarrow 1, c b}$ and $\|\cdot\|_{\infty \rightarrow \infty, c b}$ is the following:

$$
\|T\|_{1 \rightarrow 1, c b}=\left\|T^{*}\right\|_{\infty \rightarrow \infty, c b},
$$

where $T^{*}$ is the dual of $T$, satisfying $\operatorname{tr} A T(B)=\operatorname{tr} T^{*}(A) B$. We will denote $\|\cdot\|_{\infty \rightarrow \infty, c b}$ simply by $\|\cdot\|_{c b}$ when there is no risk of confusing different completely-bounded norms.

Remark 3.1. As shown in [29], the supremum in equation (2) is reached when $n$ is equal to the dimension of the space on which $T$ is acting: if $T: \mathcal{M}_{n} \rightarrow \mathcal{M}_{n}$, then $\left\|T \otimes \mathbb{1}_{n}\right\|_{1 \rightarrow 1}=\|T\|_{1 \rightarrow 1, c b}$.

The generator $\mathcal{L}$ of the semigroup $T_{t}=e^{t \mathcal{L}}$, is called a Liouvillian. All such generators can be written in the following general form, often called the Lindblad form [15, 42] (see [63]):

Proposition 3.2. $\mathcal{L}$ generates a continuous semigroup of CPTP maps if and only if it can be written in the form:

$$
\mathcal{L}(\rho)=i[\rho, H]+\sum_{j} L_{j} \rho L_{j}^{*}-\frac{1}{2} \sum_{j}\left\{L_{j}^{*} L_{j}, \rho\right\},
$$

where $H$ is a Hermitian matrix, $\left\{L_{j}\right\}_{j}$ a set of matrices called the Lindblad operators, $[\cdot, \cdot \cdot]$ denotes the commutator and $\{\cdot, \cdot\}$ the anticommutator.

We will use the term Lindbladian and Liouvillian interchangeably. Since we consider Lindbladians $\mathcal{L}$ corresponding to local dissipative dynamics, we assume that $\mathcal{L}$ is a local Lindbladian of the form:

$$
\mathcal{L}=\sum_{u \in \Lambda} \sum_{r \geqslant 0} \mathcal{L}_{u, r}, \quad \operatorname{supp} \mathcal{L}_{u, r}=b_{u}(r),
$$

where each term in the sum above can be written in the form given by equation (3).

Such a decomposition is obviously always trivially possible. We are interested in the cases in which the norms of $\mathcal{L}_{u, r}$ decay with $r$. Concretely, let us define the strength of interaction for a Lindbladian as the pair $(J, f)$ given by:

$$
J=\sup _{u, r}\left\|\mathcal{L}_{u, r}\right\|_{1 \rightarrow 1, c b}, \quad f(r)=\sup _{u} \frac{\left\|\mathcal{L}_{u, r}\right\|_{1 \rightarrow 1, c b}}{J} .
$$

The behavior of $f(r)$ as $r$ goes to infinity corresponds to various interaction regimes, listed in order of decreasing decay rate:

- finite range interaction: $f(r)$ is compactly supported;

- exponentially decaying: $f(r) \leqslant e^{-\mu r}$, for some $\mu>0$;

- quasi-local interaction: $f(r)$ decays faster than any polynomial;

- power-law decay: $f(r) \leqslant(1+r)^{-\alpha}$, for some positive $\alpha>0$. 
As we will see later, our result will apply whenever $\mathcal{L}$ has finite range, exponentially decaying, or quasilocal interactions. It will also hold in the power-law decay regime, but we will require a lower bound on the decay exponent $\alpha$, depending on the dimension of the underlying lattice. Not to overload the exposition, we will assume that $\mathcal{L}$ has finite range or exponentially decaying interactions, unless otherwise specified. The modifications needed to work with quasi-local interactions and power-law decay are presented in section 6.4 Also, we will say that functions we construct along the way are fast-decaying, if their decay rate is within the same decay class of $f(r)$ we are considering (or faster).

As shown in 62], from the spectral decomposition of $\mathcal{L}$ (and $T_{t}$ ) one can define two new CPTP maps which represent the infinite-time limit of the semigroup $T_{t}$. We will denote by $T_{\infty}$ the projector onto the subspace of stationary states (fixed points), and by $T_{\phi}$ the projector onto the subspace of periodic states. They correspond, respectively, to the kernel of $\mathcal{L}$ and to the eigenspace of purely imaginary eigenvalues of $\mathcal{L}$, which we denote $\mathcal{F}_{\mathcal{L}}$ and $\mathcal{X}_{\mathcal{L}}$, respectively. Both subspaces are invariant under $T_{t}$ : in particular, $T_{t}$ acts as the identity over $\mathcal{F}_{\mathcal{L}}$, while it is a unitary operator over $\mathcal{X}_{\mathcal{L}}$. Note, also, that both subspaces are spanned by positive operators (i.e. density matrices) [63. Prop. 6.8, Prop. 6.12]. We will denote by $T_{\phi, t}$ the composition $T_{t} \circ T_{\phi}$.

Since we plan to exploit the local structure of $\mathcal{L}$, we will often make use of the restriction of $\mathcal{L}$ to a subset of the lattice. Given $A \subset \Lambda$, we define the truncated, or localized, generator:

$$
\mathcal{L}_{A}=\sum_{b_{u}(r) \subseteq A} \mathcal{L}_{u, r}
$$

\section{$3.1 \quad$ Uniform families}

We are interested in how properties of dissipative dynamics scale with the size of the system. Hence, we are concerned with sequences of Lindbladians defined on lattices of increasing size, where all the Lindbladians in the sequence are from the same "family". To make this precise, we need to pin down how Lindbladians from the same family, but on different size lattices, are related to one-another. Our results will apply to very general sequences of Lindbladians, which we call uniform families. Before giving the precise definition, it is helpful to consider some special cases.

For local Hamiltonians on a lattice, one often considers translationally-invariant systems with various types of boundary conditions (e.g. open or periodic boundaries). There is then a natural definition of what it means to consider the same translationally-invariant Hamiltonian on different lattice sizes. Translationallyinvariant Lindbladians are an important special case of a uniform family. In this special case, all the local terms in the Lindbladian that act in the "bulk" of the lattice are the same. Another way of thinking about this is to formally consider the translationally-invariant Lindbladian $\mathcal{M}$ defined on the infinite lattice $\Gamma=\mathbb{Z}^{d}$, and then consider each member of the family to be a restriction of this infinite Lindbladian to a finite sub-lattice $\Lambda \subset \Gamma$ of some particular size:

$$
\mathcal{L}=\mathcal{M}_{\Lambda}
$$

This gives us translationally-invariant Lindbladians with open boundary condition. But of course, this is only one particular choice of boundary terms (in this case, no boundary terms at all). We are also interested in more general boundary conditions, such as periodic boundaries. So, in addition to the "bulk" interactions coming from $\mathcal{M}$, we allow additional terms that play the role of boundary conditions:

$$
\mathcal{L}=\mathcal{M}_{\Lambda}+\mathcal{L}^{\partial \Lambda} .
$$

We allow greater freedom in the boundary terms $\mathcal{L}^{\partial \Lambda}$. For one thing, they are allowed to depend on the size of the lattice $\Lambda$. But more importantly, we allow strong interactions that cross the boundary of $\Lambda$, coupling sites that would otherwise be far apart. For example, the case of periodic boundary conditions corresponds to adding interaction terms that connect opposite boundaries of $\Lambda$, as if on a torus (see Fig. 1).

Now that we have given an intuition of what a uniform family is, it is time to present the formal definition. This includes all the special cases discussed so far, but also captures much more general families of 


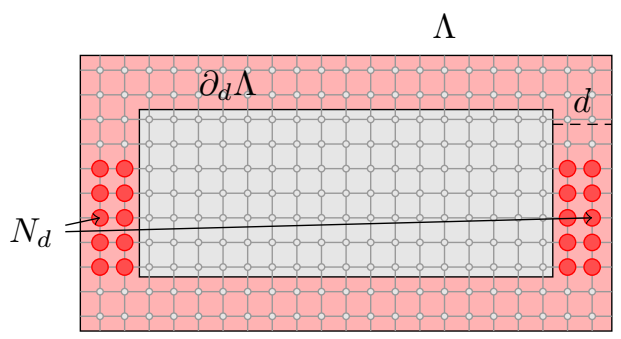

Figure 1: Partition of the lattice $\Lambda$ into the bulk and the boundary of thickness $d, \partial_{d} \Lambda$ (see Def. 3.3. The dark red regions on the boundary correspond to the interaction term $N_{d}$ coupling distant regions in $\Lambda$.

Lindbladians that are not necessarily translationally-invariant, and many other types of boundary conditions (e.g. cylindrical boundaries, or boundary terms that give the sphere topology, or terms that force fixed states on the boundary ${ }^{5}$.

Definition 3.3. Given $\Lambda \subset \Gamma$, a boundary condition with strength $(J, f)$ for $\Lambda$ is a Lindbladian $\mathcal{L}^{\partial \Lambda}=$ $\sum_{d \geqslant 1} N_{d}$, where

$$
\left\|N_{d}\right\|_{1 \rightarrow 1, c . b .} \leqslant J\left|\partial_{d} \Lambda\right| f(d)
$$

with

$$
\begin{aligned}
\partial_{d} \Lambda:= & \left\{x \in \Lambda \mid \operatorname{dist}\left(x, \Lambda^{c}\right) \leqslant d\right\}, \\
& \operatorname{supp} N_{d} \subset \partial_{d} \Lambda .
\end{aligned}
$$

Definition 3.4. A uniform family of Lindbladians $\mathcal{L}$ with strength $(J, f)$ is given by the following:

(i) infinite Lindbladian: a Lindbladian $\mathcal{M}$ on all of $\mathbb{Z}^{D}$ with strength $(J, f)$;

(ii) boundary conditions: a set of boundary conditions $\mathcal{L}^{\partial \Lambda}$, with strength $(J, f)$ and $\Lambda=b_{u}(L)$, for each $u \in \mathbb{Z}^{D}$ and $L \geqslant 0$.

We say that the family is translationally invariant if $\mathcal{M}$ is translationally invariant and $\mathcal{L}^{\partial b_{u}(L)}$ is independent of $u$.

Given a uniform family $\mathcal{L}$, we fix the following notation for evolutions defined on a subset $\Lambda$ :

$$
\begin{aligned}
\mathcal{L}^{\Lambda}=\mathcal{M}_{\Lambda} & \text { "open boundary" evolution; } \\
\mathcal{L}^{\bar{\Lambda}}=\mathcal{M}_{\Lambda}+\mathcal{L}^{\partial \Lambda} & \text { "closed boundary" evolution, }
\end{aligned}
$$

with the respective evolutions $T_{t}^{\Lambda}=\exp \left(t \mathcal{L}^{\Lambda}\right)$ and $T_{t}^{\bar{\Lambda}}=\exp \left(t \mathcal{L}^{\bar{\Lambda}}\right)$.

Remark 3.5. In the rest of the paper, we will make use of the following notation:

$$
A(s)=\{x \in \Lambda \mid \operatorname{dist}(x, A) \leqslant s\} .
$$

Since we are interested in observables whose support is not connected, we want to consider more general regions than balls: in particular, we are interested in disjoint unions of convex regions (for example, to calculate two-point correlation functions). Consider what happens to such a region $A=A_{0} \sqcup A_{1}$ when we grow it by taking $A(s)$. When $s$ becomes sufficiently large, $A_{0}(s)$ will merge with $A_{1}(s)$. At this point, $A(s)$ will not be a disjoint union of balls anymore. To avoid such complications, for $s$ large enough that disjoint balls merge, we will replace $A(s)$ by the smallest ball containing it. This will not hurt us, as $|A(s)|$ will still grow asymptotically at the same rate, which will be sufficient for our purposes.

\footnotetext{
${ }^{5}$ Or even Möbius strips, Klein bottles, and other exotic topologies.
} 


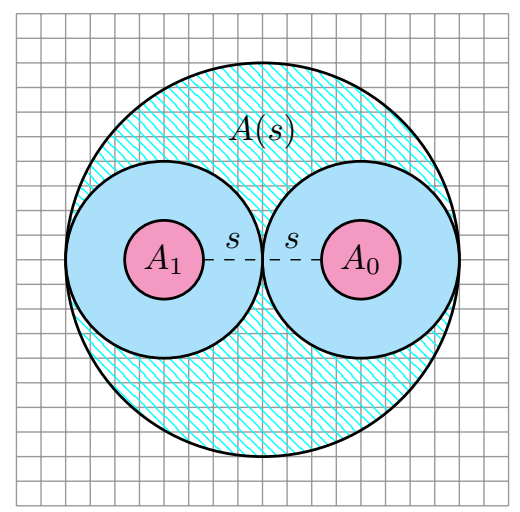

Figure 2: The convention on how to grow a region $A=A_{0} \sqcup A_{1}$.

Definition 3.6. We say that $\mathcal{L}$ has a unique fixed point if, for all $\Lambda=b_{u}(L), \mathcal{X}_{\mathcal{L}^{\bar{\Lambda}}}=\mathcal{F}_{\mathcal{L}^{\bar{\Lambda}}}=\left\{\rho_{\infty}^{\bar{\Lambda}}\right\}$. In other words, $T_{\phi}^{\bar{\Lambda}}(\rho)=T_{\infty}^{\bar{\Lambda}}(\rho)=\rho_{\infty}^{\bar{\Lambda}}$, for all density matrices $\rho$.

Note that if for all pure states $\rho$, we have $T_{t}^{\bar{\Lambda}}(\rho)>0$ (positive definite), for $t>0$, then the evolution has a unique fixed point $\rho_{\infty}>0$ (see [63, Thm. 6.7]).

We will drop the superscript from $T_{t}^{\bar{\Lambda}}$, and simply write $T_{t}$, when we consider some fixed $\Lambda \subset \Gamma$. In that case, we will refer to the number of lattice sites in $\Lambda$ as the system size.

\section{Main result}

\subsection{Assumptions for stability}

In Hamiltonian systems, the spectral gap (the difference between the two lowest energy levels) plays a crucial role in a number of settings, from defining quantum phases and phase transitions [55] to understanding the entanglement and correlations present in the system [21, 23, 25 and analyzing its stability to perturbations [10, 49]. On the other hand, it is known that for Lindbladians, the spectral gap (in this setting, the least negative real part of the non-zero eigenvalues) alone is not sufficient to fully characterize the convergence properties of the dissipative evolution 32,58 . Therefore, we will instead impose a more general requirement on the convergence of the dynamics. (The dependence of this requirement on spectral properties of $\mathcal{L}$, i.e. properties depending on the eigenvalues - like the gap - and eigenvectors - like the condition number, is an active area of research.)

Definition 4.1 (rapid mixing). Given a one-parameter semigroup of CPTP maps $T_{t}$, define the contraction of $T_{t}$ as the following quantity:

$$
\eta\left(T_{t}\right)=\frac{1}{2} \sup _{\substack{\rho \geqslant 0 \\ \operatorname{tr} \rho=1}}\left\|T_{t}(\rho)-T_{\phi, t}(\rho)\right\|_{1} .
$$

Given a family of such semigroups $\left\{T_{t}^{\alpha}\right\}_{\alpha}$, each of which is acting on $\mathcal{B}\left(H_{\alpha}\right)$ for some Hilbert space $H_{\alpha}$ of finite dimension $d_{\alpha}$, we say that it satisfies rapid mixing if there exist constants $c, \gamma, \delta>0$, such that for each $\alpha$ :

$$
\eta\left(T_{t}^{\alpha}\right) \leqslant c \log ^{\delta}\left(d_{\alpha}\right) e^{-t \gamma}
$$

We will write $\operatorname{RM}(\gamma, \delta)$ for short.

If each $H_{\alpha}$ has a tensor product structure of the type defined in equation (1), then the rapid mixing assumption can be restated as a logarithmic scaling with system size of the mixing time. Since the dimension 
of $\mathcal{H}_{\Lambda}$ is $\left(\operatorname{dim} \mathcal{H}_{x}\right)^{|\Lambda|}$, for uniform families condition 10$]$ is equivalent to:

$$
\eta\left(T_{t}^{\bar{\Lambda}}\right) \leqslant c|\Lambda|^{\delta} e^{-t \gamma} \forall \Lambda .
$$

Let us recall a result from 32 .

Theorem 4.2 (Contraction for commuting Lindbladians). Let $\left\{\mathcal{L}_{j}\right\}_{j=0}^{n}$ be a set of commuting Lindbladians. Define $\mathcal{L}=\sum_{j} \mathcal{L}_{j}$ and the corresponding evolutions $T_{t}^{j}=e^{t \mathcal{L}_{j}}$ and $T_{t}=e^{t \mathcal{L}}$. Then:

$$
\eta\left(T_{t}\right) \leqslant \sum_{j} \eta\left(T_{t}^{j}\right)
$$

In particular, consider the definition of $T_{t}^{\bar{\Lambda}}$ given in remark 3.5 for $\Lambda \subset \Gamma$ being a disjoint union of balls. Then the previous theorem implies that, if $\mathcal{L}$ is translationally-invariant and it satisfies equation (10) for each of the connected components of $\Lambda$, then it also satisfies the same equation (up to constants) for $\Lambda$.

Finally, for translationally-invariant uniform families of Lindbladians, it is sufficient to satisfy equation 10 for lattices centered at the origin: $\Lambda=b_{0}(L), L \geqslant 1$.

\subsection{Stability}

With the required assumptions laid out, we can now state our main result.

Theorem 4.3. Let $\mathcal{L}$ be a uniform family of local Lindbladians with a unique fixed point, satisfying rapid mixing (equation (11)), and consider a perturbation of the form:

$$
E^{\bar{\Lambda}}=\sum_{u \in \Lambda} \sum_{r \geqslant 0} E_{u, r}+\sum_{d \geqslant 1} E_{d}
$$

where $E_{u, r}$ is supported on $b_{u}(r)$ and each $E_{d}$ is supported on $\partial_{d} \Lambda$ (see definition 3.3) and

$$
\left\|E_{u, r}\right\|_{1 \rightarrow 1, c b} \leqslant \varepsilon e(r), \quad\left\|E_{d}\right\|_{1 \rightarrow 1, c b} \leqslant \varepsilon\left|\partial_{d} \Lambda\right| e(d),
$$

where $\varepsilon>0$ is a constant (the strength of the perturbation) and $e(r)$ is a fast-decaying function. Consider the perturbed evolution

$$
S_{t}=\exp t\left(\mathcal{L}^{\bar{\Lambda}}+E^{\bar{\Lambda}}\right)
$$

and suppose that the following assumptions hold:

(i) $E_{u, r}^{*}(\mathbb{1})=E_{d}^{*}(\mathbb{1})=0$ (or, equivalently: $\operatorname{tr} E_{u, r}\left(O_{A}\right)=\operatorname{tr} E_{d}\left(O_{A}\right)=0$, for all operators $\left.O_{A}\right)$.

(ii) $S_{t}$ is a contraction for each $t \geqslant 0$.

For an observable $O_{A}$ supported on $A \subset \Lambda$, we have for all $t \geqslant 0$ :

$$
\left\|T_{t}^{*}\left(O_{A}\right)-S_{t}^{*}\left(O_{A}\right)\right\| \leqslant c(|A|)\left\|O_{A}\right\|\left(\varepsilon+|\Lambda| \nu_{\eta}^{-1}\left(d_{A}\right)\right),
$$

where $d_{A}=\operatorname{dist}\left(A, \Lambda^{c}\right) ; \eta$ is positive and independent of $\Lambda ; \nu_{\eta}^{-1}(d) \leqslant(1+d)^{-D-1} ; c(|A|)$ is independent of $\Lambda$ and $t$, and is bounded by a polynomial in $|A|$.

Remark 4.4. Note that, for a fixed $A$, if we let $\Lambda$ grow then $d_{A}$ will increase with the linear size of $\Lambda$ and consequently $|\Lambda| \nu_{\eta}^{-1}\left(d_{A}\right)$ will vanish in the limit.

Remark 4.5. The assumptions (i)-(ii) on the perturbation $E$ are satisfied whenever $\mathcal{M}_{u, r}+E_{u, r}$ and $N_{d}+E_{d}$ (as in definition 3.4) are Lindbladians, but the theorem also covers more general perturbations. 
Remark 4.6. Since we are free to choose an $O_{A}$ with support on two non connected regions, we can apply theorem 6.7 to two-point correlation functions (or more generally $k$-point correlation functions, for fixed $k$ ) and still obtain that the error introduced by the perturbation depends linearly on the strength of the perturbation (and not on its global norm).

A set of tools already applied in the setting of classical Markov chains 16 18, 47, and recently generalized to quantum dissipative systems [31, 60], are the so-called Logarithmic Sobolev inequalities (in short, logSobolev inequalities). Introduced in a different setting to study hypercontractivity of semigroups [33], they provide the right asymptotic regime needed to satisfy the rapid mixing condition: in fact, the existence of a system size independent log-Sobolev constant implies a logarithmic scaling of the mixing time, which is exactly what is required in definition 4.1. Without going into the technical details of log-Sobolev inequalities (which can be found in $[31,60]$ ), we summarize this fact in the following corollary:

Corollary 4.7. Let $\mathcal{L}$ belong to a uniform family of translationally-invariant Lindbladians with a unique fixed point for each system size. If $\mathcal{L}$ satisfies the log-Sobolev inequality with a system-size independent constant, then the dissipative dynamics are stable, in the sense of theorem 4.3.

In particular, in [59] it was shown that product evolutions, i.e. Lindbladians that can be decomposed as a sum of independent terms $\mathcal{L}_{k}$ acting on a single subsystem, satisfy a log-Sobolev inequality with a log-Sobolev constant lower bounded by the minimum of the spectral gaps of $\mathcal{L}_{k}$ (times a factor depending on the maximum dimension of the subsystems). Moreover, the authors of [59] were able to show that Davies maps associated to a graph state Hamiltonian [26] (which are not in a product form, but can be analyzed in a similar way) and the ones associated to free-fermionic Hamiltonians have a system-size independent log-Sobolev constant.

In all such cases, corollary 4.7 implies that the evolution of local observables is stable.

\subsection{Local observables vs. global observables}

The bound in equation (13) scales with the size of the support of the observable $O_{A}$. Although the dependence is polynomial, for observables with large support the result is not useful. Still, in most realistic experiments, we are interested in the behavior of observables with fixed support and low-degree correlation functions, making the above result widely applicable. Nonetheless, one might ask more generally for a system-size independent bound on:

$$
\sup _{\rho}\left\|T_{\infty}(\rho)-S_{\infty}(\rho)\right\|_{1},
$$

where $S_{\infty}$ is the fixed-point projector for the evolution of the perturbed Lindbladian. However, this is not possible; the limitation to local observables is in some sense strict. There is no hope of finding such a bound for global observables, as the following simple example shows ${ }^{6}$

Example 4.8. Consider $N$ independent amplitude damping processes, with uniform rate $\gamma$ (which we can suppose w.l.o.g. equal to 1). This Lindbladian can be written as

$$
\mathcal{L}_{N}=\sum_{k=1}^{N} \mathbb{1}_{1 \ldots k-1} \otimes \mathcal{L}_{1} \otimes \mathbb{1}_{k+1 \ldots N}
$$

where

$$
\mathcal{L}_{1}(\rho)=|0\rangle\langle 1|\rho| 1\rangle\langle 0|-\frac{1}{2}\{\rho,|1\rangle\langle 1|\}
$$

is an amplitude damping process on a single qubit, describing the decay of the state $|1\rangle$ into $|0\rangle$ at a constant rate $\gamma=1$. This Lindabladian has gap $1 / 2$ and $e^{t \mathcal{L}_{N}}=\left(e^{t \mathcal{L}_{1}}\right)^{\otimes N}$ has mixing time of order $O(\log N)$ 32. Sec. V. C.]. The fixed point is the pure state $|0 \ldots 0\rangle\langle 0 \ldots 0|$.

\footnotetext{
${ }^{6}$ Indeed, all global stability results for quantum Linbladians we are aware of have a dependency on the total Hilbert space dimension 57.
} 
Now consider $\mathcal{L}_{1}^{\varepsilon}$, a rotation of $\mathcal{L}_{1}$, which fixes $\left|\alpha_{0}\right\rangle=\sqrt{1-\varepsilon^{2}}|0\rangle+\varepsilon|1\rangle$. We have $\left\|\mathcal{L}_{1}-\mathcal{L}_{1}^{\varepsilon}\right\|_{1 \rightarrow 1}=O(\varepsilon)$, but the new fixed point $\left|\alpha_{0}\right\rangle\left\langle\left.\alpha_{0}\right|^{\otimes N}\right.$ is almost orthogonal to the original one, since the overlap between the two is

$$
\left\langle 0 \ldots 0 \mid \alpha_{0} \ldots \alpha_{0}\right\rangle=\left\langle 0 \mid \alpha_{0}\right\rangle^{N}=\left(1-\varepsilon^{2}\right)^{N / 2} \sim e^{-N \varepsilon^{2} / 2} \rightarrow 0 \text { as } N \rightarrow \infty .
$$

This shows that, in general, there is no good bound on (14) (note that we have $\||0 \ldots 0\rangle\left\langle 0 \ldots 0|-| a_{0} \ldots \alpha_{0}\right\rangle\left\langle a_{0} \ldots \alpha_{0}\right| \|_{1} \geqslant$ $\left.1-\left|\left\langle 0 \ldots 0 \mid \alpha_{0} \ldots \alpha_{0}\right\rangle\right|^{2}\right)$ and that the dependence on the support of the observable in equation (13) cannot be improved: to see this consider the observable $O_{r}=|0 \ldots 0\rangle\left\langle\left. 0 \ldots 0\right|_{1 \ldots r}\right.$ acting on $r \leqslant N$ spins. $O_{r}$ has norm one, and

$$
O_{\infty}:=\lim _{t \rightarrow \infty} T_{t}^{*}\left(O_{r}\right)=\mathbb{1}, \quad O_{\infty}^{\varepsilon}:=\lim _{t \rightarrow \infty} T_{t}^{\varepsilon *}\left(O_{r}\right)=\left\langle 0 \mid \alpha_{0}\right\rangle^{2 r} \mathbb{1}=\left(1-\varepsilon^{2}\right)^{r} \mathbb{1} .
$$

Consequently, we have:

$$
\left\|O_{\infty}-O_{\infty}^{\varepsilon}\right\|=1-\left(1-\varepsilon^{2}\right)^{r}=r \varepsilon^{2}+o\left(\varepsilon^{2}\right) .
$$

This implies that any upper bound to $\left\|O_{\infty}-O_{\infty}^{\varepsilon}\right\|$ has to be at least linear in $r$, which is the size of the support of $O_{r}$.

\subsection{Do we need all the assumptions?}

It is reasonable to ask if the assumptions of theorem 4.3 are all necessary. We have just shown that we must necessarily consider local observables if we are to have meaningful bounds, but what about the other conditions? We will now present three examples, each consisting of a family of Lindbladiands with periodic boundary conditions, such that, in order:

- The family is uniform and translationally invariant, satisfies rapid mixing, but does not have a unique fixed point;

- The family has a unique fixed point, but is not uniform and fails to satisfy rapid mixing;

- The family (presented in appendix A has a unique fixed point, satisfies rapid mixing, but is not uniform.

All these systems will be shown to be unstable.

Example 4.9. Consider a 1D chain composed of $N$ 4-level systems, with an independent Lindbladian acting on each site, having the following Lindblad operators

$$
L_{1}=|0\rangle\left\langle 1\left|, \quad L_{2}=\right| 0\right\rangle\left\langle 3\left|, \quad L_{3}=\right| 2\right\rangle\left\langle 1\left|, \quad L_{4}=\right| 2\right\rangle\langle 3|,
$$

and denote by

$$
\mathcal{L}_{0}(\rho)=\sum_{i=1}^{4} L_{i} \rho L_{i}^{*}-\frac{1}{2}\left\{\rho, L_{i}^{*} L_{i}\right\}
$$

The global Lindbladian $\mathcal{L}_{N}$ is given by applying $\mathcal{L}_{0}$ independently on each site $k=1 \ldots N$ :

$$
\mathcal{L}_{N}=\sum_{k=1}^{N} \mathbb{1}_{1, \ldots, k-1} \otimes \mathcal{L}_{0} \otimes \mathbb{1}_{k+1, \ldots, N}
$$

Then we have that

$$
\mathcal{L}_{0}(|i\rangle\langle j|)= \begin{cases}0 & \text { if } i=j \in\{0,2\} \\ |0\rangle\langle 0|+| 2\rangle\langle 2|-2| i\rangle\langle j| & \text { if } i=j \in\{1,3\} \\ -\left[\chi_{\{1,3\}}(i)+\chi_{\{1,3\}}(j)\right]|i\rangle\langle j| & \text { if } i \neq j .\end{cases}
$$


Diagonal states of the form $|i\rangle\langle i|$ evolve according to the classical Markov process embedded in the Lindbladian, while off-diagonal elements $|i\rangle\langle j|$ evolve as

$$
T_{t}(|i\rangle\langle j|)=\exp \left(-t\left[\chi_{\{1,3\}}(i)+\chi_{\{1,3\}}(j)\right]\right)|i\rangle\langle j| ;
$$

where $\chi_{\{1,3\}}$ denotes the indicator function of the set $\{1,3\}$. This implies that the space of fixed points $\mathcal{F}_{\mathcal{L}_{0}}$ is given by $\operatorname{span}\{|0\rangle\langle 0|| 2\rangle,\langle 2|| 0\rangle,\langle 2|| 2\rangle,\langle 0|\}$. Since $\mathcal{L}_{0}$ has gap equal to 1 , theorem 4.2 implies that $\mathcal{L}_{N}$ satisfies rapid mixing. $\mathcal{L}_{N}$ forms a uniform family, but it does not satisfy the unique fixed point condition.

Consider now the following additional Lindbladian

$$
\mathcal{E}_{0}(\rho)=\frac{2}{N}\left[|0\rangle\langle 2|\rho| 2\rangle\langle 0|-\frac{1}{2}\{\rho,|2\rangle\langle 2|\}\right] .
$$

Then, we have:

$$
\left(\mathcal{L}_{0}+\mathcal{E}_{0}\right)(|i\rangle\langle j|)= \begin{cases}0 & \text { if } i=j=0 \\ |0\rangle\langle 0|+| 2\rangle\langle 2|-2| i\rangle\langle j| & \text { if } i=j=1,3 \\ \frac{2}{N}(|0\rangle\langle 0|-| i\rangle\langle j|) & \text { if } i=j=2 \\ -\left(\chi_{\{1,3\}}(i)+\chi_{\{1,3\}}(j)+\frac{\chi_{\{i, j\}}(2)}{N}\right)|i\rangle\langle j| & \text { if } i \neq j .\end{cases}
$$

Again, this implies that $\mathcal{F}_{\mathcal{L}_{0}+\mathcal{E}_{0}}=\{|0\rangle\langle 0|\}$. Consequently $\mathcal{L}_{N}+\mathcal{E}_{N}$ has a unique fixed point. It is not a uniform family, and it does not satisfy rapid mixing, as it is not even globally gapped. To see this, note that for $\sigma=|200 \ldots 0\rangle\langle 200 \ldots 0|-| 020 \ldots 0\rangle\langle 020 \ldots 0|$ :

$$
\left(\mathcal{L}_{N}+\mathcal{E}_{N}\right)(\sigma)=-\frac{2}{N} \sigma .
$$

Analogously, $\mathcal{L}_{N}+\mathcal{E}_{N}^{*}$ satisfies the same conditions as $\mathcal{L}_{N}+\mathcal{E}_{N}$, but the unique fixed point is now $|2 \ldots 2\rangle\langle 2 \ldots 2|$.

All three systems described above are unstable, since we can transform one into the other by applying a perturbation of order $O(1 / N)$, yet the fixed points of $\mathcal{L}_{N}+\mathcal{E}_{N}$ and $\mathcal{L}_{N}+\mathcal{E}_{N}^{*}$ are locally orthogonal (while $\mathcal{L}_{N}$ has both of them as fixed points).

\subsection{Relaxations of the rapid mixing condition}

In the case of finite range or exponentially decaying interactions, the proof of theorem 4.3 still holds if we relax equation (11) by requiring only a polynomial decay in time, i.e. a bound of the form

$$
\eta\left(T_{t}^{\bar{\Lambda}}\right) \leqslant c|\Lambda|^{\delta} \gamma(t),
$$

if $\gamma(t)$ is a fast enough decaying function, where the threshold decay rate is determined by system-size independent costants (such as the Lieb-Robinson bound constants and the geometrical dimension of the underlying lattice structure).

Determining the precise value of such threshold requires an argument similar to the one given for the case of power-law decaying interactions in section 6.4, and is presented in section 6.5.

\section{Toolbox for the proof}

Before presenting the proof of theorem 4.3 , we need to introduce some useful tools. We present them in full generality, including the case of power-law decay of interactions, without restricting here to exponentially decaying interactions. 


\subsection{Lieb-Robinson bounds for Lindbladian evolution}

We first recall a generalization of Lieb-Robinson bounds to non-Hamiltonian evolution, due to [54] and [50], which we use to derive a number of useful tools that allow us to approximate the support of an evolving observable with a finite set which grows linearly in time. The following condition is sufficient for the bounds to hold.

Assumption 5.1 (Lieb-Robinson condition). Let $\mathcal{L}=\sum_{u, r} \mathcal{L}_{u, r}$ be a local Lindbladian. There exist positive constants $\mu$ and $v$, such that:

$$
\sup _{x \in \Lambda} \sum_{u \in \Lambda} \sum_{r \geqslant \operatorname{dist}(u, x)}\left\|\mathcal{L}_{u, r}\right\|_{1 \rightarrow 1, c b}\left|b_{u}(r)\right| \nu_{\mu}(r) \leqslant \frac{v}{2}<\infty ;
$$

where $\nu_{\mu}(\cdot)$ is one of the following:

$$
\begin{aligned}
\nu_{\mu}(r) & =e^{\mu r}, \\
\nu_{\mu}(r) & =(1+r)^{\mu} .
\end{aligned}
$$

Note that both functions are submultiplicative, in the sense that $\nu_{\mu}(r+s) \leqslant \nu_{\mu}(r) \nu_{\mu}(s)$. Moreover, $\nu_{a}(r)^{b}=$ $\nu_{a b}(r)$.

The constant $v$ is called the Lieb-Robinson velocity of $\mathcal{L}$, while the reciprocal function $\nu_{\mu}^{-1}(r)=1 / \nu_{\mu}(r)$ is called the Lieb-Robinson decay of $\mathcal{L}$.

Note that if $\mathcal{L}$ has interaction strength $(J, f)$, then condition 16$)$ can be replaced by:

$$
J \sup _{x, y \in \Lambda} \sum_{n \geqslant 0}\left|b_{x}(r) \backslash b_{x}(r-1)\right| \sum_{r \geqslant n} f(r)\left|b_{y}(r)\right| \nu_{\mu}(r) \leqslant \frac{v}{2}<\infty .
$$

Since our systems are embedded in the lattice $\mathbb{Z}^{D}$, we have that $v<\infty$, as long as:

$$
\sum_{n \geqslant 0} n^{D-1} F_{\mu}(n)<\infty, \quad F_{\mu}(n):=\sum_{r \geqslant n} r^{D} f(r) \nu_{\mu}(r) .
$$

Remark 5.2. Condition (LR-1) is satisfied when $\mathcal{L}$ has finite-range or exponentially decaying interactions, while condition (LR-2 is satisfied when $\mathcal{L}$ has quasi-local interactions. If $\mathcal{L}$ has interactions decaying as a power-law with exponent $\alpha$, then condition (LR-2) is satisfied whenever $\alpha>2 D+1$ (by choosing $\mu<\alpha-(2 D+1))$.

Theorem 5.3 (Lieb-Robinson bound). Suppose $\mathcal{L}$ is a local Lindbladian satisfying assumption 5.1. Let $O_{X}$ be an observable supported on $X \subset \Lambda$, and denote by $O_{X}(t)=T_{t}^{*}\left(O_{X}\right)$ its evolution under $\mathcal{L}$. Let $K: \mathcal{A}_{Y} \rightarrow \mathcal{A}_{Y}$ be a super-operator supported on $Y \subset \Lambda$ which vanishes on $\mathbb{1}$. Then, the following bound holds [50, 54]:

$$
\|K(O(t))\| \leqslant\|K\|_{\infty \rightarrow \infty, c b}\left\|O_{X}\right\| C(X, Y) \frac{\left(e^{v t}-1\right)}{\nu_{\mu}(\operatorname{dist}(X, Y))},
$$

where $C(X, Y)=\min (|X|,|Y|)$.

From now on, we will only consider Lindbladians which satisfy equation 117 with either of the two possible assumptions on $\nu_{\mu}(\cdot)$.

Lemma 5.4 (Comparing different dynamics). Let $\mathcal{L}_{1}$ and $\mathcal{L}_{2}$ be two local Lindbladians, and suppose $\mathcal{L}_{2}$ has Lieb-Robinson speed and decay bounded by $v$ and $\nu_{\mu}^{-1}$. Consider an operator $O_{X}$ supported on $X \subset \Lambda$, and denote by $O_{i}(t)$ its evolution under $\mathcal{L}_{i}, i=1,2$. Suppose that $\mathcal{L}_{1}-\mathcal{L}_{2}=\sum_{r \geqslant 0} M_{r}$, where $M_{r}$ is a superoperator supported on $Y_{r}$ which vanishes on $\mathbb{1}$, and $\operatorname{dist}\left(X, Y_{r}\right) \geqslant r$. Then the following holds:

$$
\left\|O_{1}(t)-O_{2}(t)\right\| \leqslant\left\|O_{X}\right\||X| \frac{e^{v t}-v t-1}{v} \sum_{r=0}^{\infty}\left\|M_{r}\right\|_{1 \rightarrow 1, c b} \nu_{\mu}^{-1}(r) .
$$


Proof. Let $h(t)=O_{1}(t)-O_{2}(t)$. Calculating its derivative, we obtain

$$
h^{\prime}(t)=\mathcal{L}_{1}^{*} O_{1}(t)-\mathcal{L}_{2}^{*} O_{2}(t)=\mathcal{L}_{1}^{*} h(t)+\left(\mathcal{L}_{1}^{*}-\mathcal{L}_{2}^{*}\right) O_{2}(t) .
$$

Since $h(0)=0$, this differential equation for $h(t)$ has solution

$$
\begin{aligned}
h(t)=O_{1}(t)-O_{2}(t) & =\int_{0}^{t} e^{(t-s) \mathcal{L}_{1}^{*}}\left(\mathcal{L}_{1}^{*}-\mathcal{L}_{2}^{*}\right) O_{2}(s) \mathrm{d} s \\
& =\sum_{r \geqslant 0} \int_{0}^{t} e^{(t-s) \mathcal{L}_{1}^{*}} M_{r}^{*} O_{2}(s) \mathrm{d} s,
\end{aligned}
$$

giving us a useful integral representation for $O_{1}(t)-O_{2}(t)$. From this, we obtain the estimate

$$
\left\|O_{1}(t)-O_{2}(t)\right\| \leqslant \sum_{r \geqslant 0} \int_{0}^{t}\left\|M_{r}^{*} O_{2}(s)\right\| \mathrm{d} s,
$$

where we have used the fact that $e^{t \mathcal{L}_{1}^{*}}$ is a contraction with respect to $\|\cdot\|_{\infty}$ for each $t \geqslant 0$.

We can now apply the Lieb-Robinson bound (equation (19) to each of the terms in the sum in the previous estimate, to obtain:

$$
\left\|O_{1}(t)-O_{2}(t)\right\|
$$

$$
\leqslant \sum_{r \geqslant 0}\left\|M_{r}\right\|_{1 \rightarrow 1, c b}\left\|O_{X}\right\| C\left(X, Y_{r}\right) \nu_{\mu}^{-1}\left(\operatorname{dist}\left(X, Y_{r}\right)\right) \int_{0}^{t}\left(e^{v s}-1\right) \mathrm{d} s
$$

which implies the claimed bound.

A particular application of the previous lemma is when $\mathcal{L}_{2}$ is a restriction of $\mathcal{L}_{1}$ onto a smaller region. Since this case occurs frequently, and is of particular interest, we state it as a separate lemma:

Lemma 5.5 (Localizing the evolution). Let $O_{A}$ be an observable supported on a finite $A \subset \Lambda$. Denote by $O_{A}(t)=T_{t}^{*}\left(O_{A}\right)$ its evolution under a local Lindbladian $\mathcal{L}$ with strength $(J, f)$. Given $r>0$, denote by $O_{A}(r ; t)$ its evolution under the localized Lindbladian $\mathcal{L}_{A(r)}$.

Then, the following bound holds:

$$
\left\|O_{A}(t)-O_{A}(r ; t)\right\| \leqslant\left\|O_{A}\right\||A| J \frac{e^{v t}-1-v t}{v} \nu_{\beta}^{-1}(r),
$$

where $\nu_{\beta}^{-1}(r)$ decays exponentially if $\mathcal{L}$ satisfies condition (LR-1), while decays as $(1+r)^{-\beta}$ if $\mathcal{L}$ satisfies condition $(\overline{\mathrm{LR}-2})$. In this case, if we denote by $\alpha$ the decay rate of $\mathcal{L}$, then $\beta$ is given by:

$$
\beta= \begin{cases}\alpha-3 D & \text { if } \alpha \geqslant 5 D-1 \\ \frac{1}{2}(\alpha-D-1) & \text { if } \alpha \leqslant 5 D-1\end{cases}
$$

Proof. First, let us decompose $\mathcal{L}-\mathcal{L}_{A(r)}$ as a telescoping sum

$$
\mathcal{L}-\mathcal{L}_{A(r)}=\sum_{l \geqslant r} \mathcal{L}_{A(l+1)}-\mathcal{L}_{A(l)} .
$$

Since each element in the sum is the difference between restrictions on different subsets of the same global Lindbladian, it is easy to explicitly write their difference

$$
\mathcal{L}_{A(l+1)}-\mathcal{L}_{A(l)}=\sum_{\delta=0}^{l+1} \sum_{\operatorname{dist}(u, A)=\delta} \mathcal{L}_{u}(l+1-\delta) .
$$


We group the terms in the sum by their distance from $A$ : Let

$$
d=\operatorname{dist}\left(A, b_{u}(l+1-\delta)\right)=\max \{0,2 \delta-l-1\}
$$

and

$$
\begin{aligned}
& M_{0}=\sum_{l \geqslant r} \sum_{\delta=0}^{\frac{l+1}{2}} \sum_{\operatorname{dist}(u, A)=\delta} \mathcal{L}_{u}(l+1-\delta) ; \\
& M_{d}=\sum_{\substack{l \geqslant r \\
\operatorname{dist}(u, A)=\delta \\
\delta=\frac{l+1+d}{2}}} \mathcal{L}_{u}(l+1-\delta) .
\end{aligned}
$$

Then, we can write:

$$
\sum_{d \geqslant 0} M_{d}=\mathcal{L}-\mathcal{L}_{A(r)} ; \quad \operatorname{dist}\left(A, \operatorname{supp} M_{d}\right)=d .
$$

Applying lemma 5.4 , we obtain:

$$
\left\|O_{A}(t)-O_{A}(r ; t)\right\| \leqslant\left\|O_{A}\right\||A| J \frac{e^{v t}-1-v t}{v} \zeta(r) ;
$$

where, by denoting $q(l)=|A(l) \backslash A(l-1)|, \zeta(r)$ is the following:

$$
\begin{aligned}
\zeta(r)=\frac{1}{J} \sum_{d \geqslant 0}\left\|M_{d}\right\|_{1 \rightarrow 1, c b} \nu_{\mu}^{-1}(d) \leqslant & \\
& \sum_{l \geqslant r} \sum_{\delta=0}^{\frac{l+1}{2}} q(\delta) f(l+1-\delta)+\sum_{\delta=\frac{l+1}{2}}^{l+1} q(\delta) f(l+1-\delta) \nu_{\mu}^{-1}(2 \delta-l-1) .
\end{aligned}
$$

If $\delta \geqslant(l+1) / 2$, since $\nu_{\mu}(\cdot)$ is submultiplicative, we have:

$$
\nu_{\mu}(\delta) \leqslant \nu_{\mu}(l+1-\delta) \nu_{\mu}(2 \delta-l-1) .
$$

Otherwise, since $\nu_{\mu}(\cdot)$ is increasing, we have that $\nu_{\mu}(\delta) \leqslant \nu_{\mu}(l+1-\delta)$. Plugging these inequalities in the above sum, we get:

$$
\zeta(r) \leqslant \sum_{l \geqslant r} \sum_{\delta=0}^{l+1}\left[q(\delta) \nu_{\mu}^{-1}(\delta)\right]\left[f(l+1-\delta) \nu_{\mu}(l+1-\delta)\right] .
$$

Since $f$ satisfies equation (17), which in particular implies

$$
\sum_{\delta \geqslant 0} f(\delta) \nu_{\mu}(\delta)\left|b_{0}(\delta)\right|<\infty
$$

then the sequence $f(\delta) \nu_{\mu}(\delta)$ is decreasing. We distinguish two cases: If $\nu_{\mu}$ is of the type (LR-1), then the decay of $f(\delta) \nu_{\mu}(\delta)$ is exponential. Since $q(\delta)$ grows polynomially, $q(\delta) \nu_{\mu}^{-1}(\delta)$ is exponentially decaying. Then, the convolution of the two sequences, which is exactly:

$$
\sum_{\delta=0}^{l+1}\left[q(\delta) \nu_{\mu}^{-1}(\delta)\right]\left[f(l+1-\delta) \nu_{\mu}(l+1-\delta)\right]
$$

is exponentially decaying too, which implies an exponential decay rate for $\zeta(r)$. Thus, there exists some $\beta>0$ such that $\zeta(r) \leqslant \nu_{\beta}^{-1}(r)$, and this concludes the proof for the case of exponential decay. Let us 
suppose now that $\nu_{\mu}$ is of type LR-2. Then, $f(\delta) \nu_{\mu}(\delta)$ decays as $(1+\delta)^{\mu-\alpha}$, while $q(\delta) \nu_{\mu}^{-1}(\delta)$ decays as $(1+\delta)^{D-1-\mu}$. This implies 7 that their convolution decays as $(1+l)^{-\min (\alpha-\mu, \mu-D+1)}$ and thus

$$
\zeta(r) \leqslant c(1+r)^{-\min (\alpha-\mu-1, \mu-D)}=\nu_{\beta}^{-1}(r) .
$$

Recalling that condition (LR-2 requires $\mu<\alpha-(2 D+1)$, a simple calculation shows that the above decay rate is maximized for

$$
\mu<\min \left(\alpha-2 D-1, \frac{\alpha+D-1}{2}\right)
$$

which gives the claimed formula for $\beta$.

Another specialization of lemma 5.4 similar in spirit to the one just presented, is when we compare the evolution of local observables under $\mathcal{L}^{A(r)}$ and $\mathcal{L}^{\overline{A(r)}}$, as defined in definition 3.4 .

Lemma 5.6. Let $O_{A}$ be an observable supported on $A \subset \Lambda$. Given $r>0$, it holds that

$$
\left\|T_{t}^{* \overline{A(r)}}\left(O_{A}\right)-T_{t}^{* A(r)}\left(O_{A}\right)\right\| \leqslant\left\|O_{A}\right\||A| J \frac{e^{v t}-1-v t}{v} \nu_{\beta}^{-1}(r) .
$$

Proof. Without loss of generality, we consider the case of $A(r)$ being a convex set. By construction, $\mathcal{L}^{\overline{A(r)}}-$ $\mathcal{L}^{A(r)}=\mathcal{L}^{\partial A(r)}$, and $\mathcal{L}^{\partial A(r)}=\sum_{d \geqslant 1} N_{d}$, where each $N_{d}$ acts on sites that are closer than $d$ to the border of $A(r)$. We group these terms by their distance from $A$. Let $k=\frac{1}{2} \operatorname{diam} A$ and set:

$$
\begin{aligned}
& M_{0}=\sum_{i=0}^{k} N_{r+1+i} \\
& M_{j}=N_{r+1-j}, \quad j=1 \ldots r .
\end{aligned}
$$

It is easy to see that $\operatorname{dist}\left(A, \operatorname{supp} M_{j}\right)=j$. By applying lemma 5.4 we have that:

$$
\left\|T_{t}^{* \overline{A(r)}}\left(O_{A}\right)-T_{t}^{* A(r)}\left(O_{A}\right)\right\| \leqslant\left\|O_{A}\right\||A| \frac{e^{v t}-1-v t}{v} \sum_{j=0}^{r}\left\|M_{j}\right\|_{1 \rightarrow 1, c . b .} \nu_{\mu}^{-1}(j) .
$$

We are left to prove that the sum appearing on the r.h.s. is fast-decaying in $r$. From definition 3.4 it follows that for $j>0$ :

$$
\left\|M_{j}\right\|_{1 \rightarrow 1, c . b .} \leqslant J\left|\partial_{r-j} A(r)\right| f(r+1-j)=J|A(r) \backslash A(j)| f(r+1-j),
$$

while for $j=0$ :

$$
\left\|M_{0}\right\|_{1 \rightarrow 1, c . b .} \leqslant \sum_{i=0}^{k} J\left|\partial_{r+i} A(r)\right| f(r+1+i)
$$

Setting $h_{m, n}=\left|b_{0}(m) \backslash b_{0}(n)\right|$, we have that:

$$
\sum_{j=0}^{r}\left\|M_{j}\right\|_{1 \rightarrow 1, c . b .} \nu_{\mu}^{-1}(j) \leqslant J \zeta(r)
$$

where

$$
\zeta(r):=\sum_{i=0}^{k} h_{r+k, k-i} f(r+1+i)+\sum_{j=1}^{r} h_{r+k, k+j} f(r+1-j) \nu_{\mu}^{-1}(j) .
$$

${ }^{7}$ Consider two positive decreasing sequences $\left(x_{n}\right)$ and $\left(y_{n}\right)$. Since $0<p<1$ implies that $(x+y)^{p} \leqslant x^{p}+y^{p}$, it holds that $(x * y)_{n}^{p} \leqslant \sum_{k} x_{k}^{p} y_{n-k}^{p}=\left(x^{p} * y^{p}\right)_{n}$. 
An argument similar to the one in the proof of lemma 5.5 shows that $\zeta(r)$ is fast-decaying. Indeed, $h_{r+k, k-i} f(r+1+i)$ scales asymptotically as $r^{D} f(r)$, while $h_{r+k, k+j} f(r+1-j)$ scales as $(r-j)^{D} f(r+1-j)$. If $\mathcal{L}$ satisfies (LR-1), then $\zeta(r)$ will be exponentially decaying, with rate $\min (\alpha, \mu)-1=\mu-1$.

If otherwise $\mathcal{L}$ satisfies $([\mathrm{LR}-2)$, then $\zeta(r)$ has a polynomial decay, with rate $\min (\alpha-D, \mu)-1=\mu-1$. In both cases, then:

$$
\zeta(r) \leqslant \nu_{\mu-1}^{-1}(r) .
$$

Notice that the constant $\beta$ defined in lemma 5.5 is smaller than $\mu-1$.

\section{$5.2 \quad$ Local rapid mixing}

The rapid mixing condition implies a local version of mixing that will be a useful tool for the proof of theorem 4.3 We state its definition here.

Definition 5.7 (Local rapid mixing). Take $A \subset \Lambda$, and define the contraction of $T_{t}$ relative to $A$ as

$$
\begin{aligned}
\eta^{A}\left(T_{t}\right) & :=\sup _{\substack{\rho \geqslant 0 \\
\operatorname{tr} \rho=1}}\left\|\operatorname{tr}_{A^{c}}\left[T_{t}(\rho)-T_{\phi, t}(\rho)\right]\right\|_{1} \\
= & \sup _{\substack{\rho \geqslant 0 \\
\operatorname{tr} \rho=1 \| O_{A} \in \mathcal{A}_{A}}} \sup _{\substack{O_{A}\\
}} \operatorname{tr}\left(O_{A}\left[T_{t}(\rho)-T_{\phi, t}(\rho)\right]\right) \\
& =\sup _{\substack{\rho \geqslant 0 \\
\operatorname{tr} \rho=1}} \sup _{A \in \mathcal{A}_{A}} \operatorname{tr}\left(\rho\left[T_{t}^{*}\left(O_{A}\right)-T_{\phi, t}^{*}\left(O_{A}\right)\right]\right) .
\end{aligned}
$$

We say that $\mathcal{L}$ satisfies local rapid mixing if, for each $A \subset \Lambda$, we have that

$$
\eta^{A}\left(T_{t}\right) \leqslant k(|A|) e^{-\gamma t}
$$

where $k(r)$ grows polynomially in $r, \gamma>0$ and all the constants appearing above are independent of the system size.

Remark 5.8. It follows from the definition that $\eta^{A}\left(T_{t}\right) \leqslant \eta^{B}\left(T_{t}\right)$ whenever $A \subset B$. In particular, $\eta^{A}\left(T_{t}\right) \leqslant$ $\eta\left(T_{t}\right)$.

Note that, in contrast with definition 4.1 the quantity $\eta^{A}\left(T_{t}\right)$ depends on the evolution on the whole system $\Lambda$, and not just on the subset $A$. Thus local rapid mixing is a very strong condition: the term $k(r)$ appearing in equation 29) only depends on the support of $A$, so the local mixing time (i.e. the time it takes for the reduced density matrix on the subset $A$ to converge) is required to be independent of system size.

Example 5.9. A simple dissipative system satisfying definition 5.7 is the tensor product of amplitude damping channels acting (with the same rate) on different qubits. Note that, though it might seem a trivial example, there are interesting dissipative systems of this form: among others, dissipative preparation of graph states 32 can be brought into this form by a non-local unitary rotation (which of course does not change the convergence rates).

\section{Proof of main result}

We are now ready to prove our main resul, theorem 4.3 The proof proceeds in three steps. First, we show that the assumptions of theorem 4.3 imply that the fixed points of $\mathcal{L}^{\bar{\Lambda}}$ for different $\Lambda$ are locally indistinguishable. Then, we prove that rapid mixing implies local rapid mixing. Finally, we show how local rapid mixing and the uniqueness of the initial fixed point imply the desired stability result. 


\subsection{Step 1: closeness of the fixed points}

Topological quantum order (TQO), namely the property of certain orthogonal quantum states to be locally indistinguishable from each other, is a widely studied property of ground state subspaces in the Hamiltonian setting. In the dissipative setting on the other hand, where the concept of ground states is no longer applicable, one may define the analogous concept for periodic states of Lindbladians. Below we describe the concept of Local Topological Quantum Order (LTQO) [49], which extends the concept of TQO to the invariant subspace (periodic states) of local restrictions of the global Lindbladian.

We note that, in contrast to the Hamiltonian case, in order to prove the desired stability result we do not require extra assumptions like LTQO, or frustration-freeness. Indeed, we show in this section that rapid mixing implies LTQO and a property similar to frustration-freeness. These properties will play a role in the proof of stability, via lemma 6.4

Definition 6.1 (Local Topological Quantum Order (LTQO)). Consider a Lindbladian $\mathcal{L}$. Take a convex set $A \subset \Lambda$ and let $A(\ell)=\{x \in \Lambda \mid \operatorname{dist}(x, A) \leqslant \ell\}$. Given two states $\rho_{i} \in \mathcal{X}_{\mathcal{L}_{A(\ell)}}, i=1,2$, consider their reduced density matrices on $A$ :

$$
\rho_{i}^{A}=\operatorname{tr}_{A(\ell) \backslash A} \rho_{i}, \quad i=1,2 .
$$

We say that $\mathcal{L}$ has local topological quantum order (LTQO) if for each $\ell \geqslant 0$ :

$$
\left\|\rho_{1}^{A}-\rho_{2}^{A}\right\|_{1} \leqslant p(|A|) \Delta_{0}(\ell)
$$

where $\Delta_{0}(\ell)$ is a fast-decaying function, and $p(\cdot)$ is a polynomial.

As a first step in the proof, we will show that the conditions of theorem 4.3 imply that the fixed point of $T_{t}$, the fixed point of $T_{t}^{\bar{A}}$ and the periodic points of $T_{t}^{A}$ are difficult to distinguish locally, in the same spirit as the LTQO condition.

Lemma 6.2. Let $\mathcal{L}$ be a uniform family satisfying condition (11), and suppose each $\mathcal{L}^{\bar{A}}$ has a unique fixed point and no other periodic points. Let $O_{A}$ be an observable supported on $A \subset \Lambda, \rho$ a periodic point of $T_{t}^{A(s)}$ and $\rho_{\infty}^{s}$ the unique fixed point of $T_{t}^{\overline{A(s)}}$. Then, we have

$$
\left|\operatorname{tr} O_{A}\left(\rho-\rho_{\infty}^{s}\right)\right| \leqslant\left\|O_{A}\right\|\left(\frac{J}{v}|A|+c|A|^{\delta}\right) \Delta_{0}(s),
$$

where

$$
\Delta_{0}(s)=(|A(s)| /|A|)^{\delta v /(v+\gamma)} \nu_{\beta^{\prime}}^{-1}(s), \quad \beta^{\prime}=\beta \gamma /(v+\gamma),
$$

with $c, \gamma, \delta$ the constants defined in the rapid mixing condition $R M(\gamma, \delta), \beta$ the rate defined in lemma 5.6 and $v$ the Lieb-Robinson velocity.

Proof. Fix a $t:=t(s) \geqslant 0$, to be determined later. Since $T_{t}^{A(s)}$ acts on its space of periodic points as a unitary evolution, there exists a periodic point of $\mathcal{L}^{A(s)}, \rho^{\prime}$, such that $\rho=T_{t}^{A(s)}\left(\rho^{\prime}\right)$. Then, by the triangle inequality, we have:

$$
\left.\left|\operatorname{tr} O_{A}\left(\rho-\rho_{\infty}^{s}\right)\right| \leqslant \mid \operatorname{tr} O_{A}\left[T_{t}^{A(s)}-T_{t}^{\overline{A(s)}}\right]\left(\rho^{\prime}\right)\right)|+| \operatorname{tr} O_{A}\left(T_{t}^{\overline{A(s)}}\left(\rho^{\prime}\right)-\rho_{\infty}^{s}\right) \mid .
$$

The first term is bounded by lemma 5.6 , since

$$
\operatorname{tr} O_{A}\left(T_{t}^{A(s)}\left(\rho^{\prime}\right)-T_{t}^{\overline{A(s)}}\left(\rho^{\prime}\right)\right)=\operatorname{tr} \rho^{\prime}\left(T_{t}^{* A(s)}\left(O_{A}\right)-T_{t}^{* \overline{A(s)}}\left(O_{A}\right)\right)
$$

and

$$
\left|\operatorname{tr} \rho^{\prime}\left(T_{t}^{* A(s)}\left(O_{A}\right)-T_{t}^{* \overline{A(s)}}\left(O_{A}\right)\right)\right| \leqslant\left\|\rho^{\prime}\right\|_{1}\left\|T_{t}^{* A(s)}\left(O_{A}\right)-T_{t}^{* \overline{A(s)}}\left(O_{A}\right)\right\|_{\infty}
$$


The second term is bounded using the rapid mixing condition on $T_{t}^{\overline{A(s)}}$. By putting the two bounds together, we obtain

$$
\left|\operatorname{tr} O_{A}\left(\rho-\rho_{\infty}^{s}\right)\right| \leqslant\left\|O_{A}\right\||A| \frac{J}{v} e^{v t} \nu_{\beta}^{-1}(s)+\left\|O_{A}\right\| c|A(s)|^{\delta} e^{-\gamma t} .
$$

Setting $p(s)=(|A(s)| /|A|)^{\delta}$ and choosing $t(s)$ such that

$$
e^{v t(s)} \nu_{\beta}^{-1}(s)=p(s) e^{-\gamma t(s)},
$$

we have that $t(s)=\ln \left(\nu_{\beta}(s) \cdot p(s)\right)^{1 /(v+\gamma)}$. Under such choice, it holds that

$$
e^{-\gamma t(s)}=\left(\nu_{\beta}(s) p(s)\right)^{-\gamma /(v+\gamma)}=\nu_{\beta^{\prime}}^{-1}(s) p(s)^{-\gamma /(v+\gamma)},
$$

where $\beta^{\prime}=\beta \gamma /(v+\gamma)$. Defining

$$
\Delta_{0}(s):=(|A(s)| /|A|)^{\delta v /(v+\gamma)} \nu_{\beta^{\prime}}^{-1}(s),
$$

concludes the proof.

Corollary 6.3 (LTQO). Under the assumptions of lemma 6.2, the Lindbladian $\mathcal{L}^{\bar{\Lambda}}$ satisfies LTQO (definition 6.1) for all $\Lambda$.

Proof. Take $A \subset \Lambda$, and $s \geqslant 0$. Let $\rho_{1}$ and $\rho_{2}$ be two periodic points of $T_{t}^{A(s)}$. Then, by the triangle inequality, we have that:

$$
\begin{aligned}
\left|\operatorname{tr} O_{A}\left(\rho_{1}-\rho_{2}\right)\right| \leqslant\left|\operatorname{tr} O_{A}\left(\rho_{1}-\rho_{\infty}^{s}\right)\right|+\left|\operatorname{tr} O_{A}\left(\rho_{\infty}^{s}-\rho_{2}\right)\right| \leqslant & \leqslant 2\left\|O_{A}\right\|\left(\frac{J}{v}|A|+c|A|^{\delta}\right) \Delta_{0}(s) .
\end{aligned}
$$

Since $\left\|\rho_{1}^{A}-\rho_{2}^{A}\right\|_{1}=\sup _{\left\|O_{A}\right\|=1}\left|\operatorname{tr} O_{A}\left(\rho_{1}-\rho_{2}\right)\right|$, the result follows immediately.

Lemma 6.4. Under the same notation and assumptions of lemma 6.2, we have the following bound for $\rho_{\infty}$ the unique fixed point of $T_{t}$ :

$$
\sup _{\left\|O_{A}\right\|=1}\left|\operatorname{tr} O_{A}\left(\rho_{\infty}-\rho_{\infty}^{s}\right)\right| \leqslant\left\|O_{A}\right\|\left(\frac{J}{v}|A|+c|A|^{\delta}\right) \Delta_{0}(s) .
$$

Proof. By the triangle inequality:

$$
\left.\mid \operatorname{tr} O_{A}\left(\rho_{\infty}-\rho_{\infty}^{s}\right)\right)|\leqslant| \operatorname{tr} O_{A}\left(\rho_{\infty}-T_{t}^{\overline{A(s)}}\left(\rho_{\infty}\right)|+| \operatorname{tr} O_{A}\left(T_{t}^{\overline{A(s)}}\left(\rho_{\infty}\right)-\rho_{\infty}^{s}\right) \mid .\right.
$$

The first term on the right can be bounded using lemmas 5.5 and 5.6 along with $T_{t}\left(\rho_{\infty}\right)=\rho_{\infty}$ :

$$
\begin{gathered}
\left|\operatorname{tr} O_{A}\left(T_{t}\left(\rho_{\infty}\right)-T_{t}^{\overline{A(s)}}\left(\rho_{\infty}\right)\right)\right|=\left|\operatorname{tr} \rho_{\infty}\left(T_{t}^{*}\left(O_{A}\right)-T_{t}^{* \overline{A(s)}}\left(O_{A}\right)\right)\right| \\
\leqslant\left\|\rho_{\infty}\right\|_{1}\left(\left\|T_{t}^{*}\left(O_{A}\right)-T_{t}^{* A(s)}\left(O_{A}\right)\right\|_{\infty}+\left\|T_{t}^{* A(s)}\left(O_{A}\right)-T_{t}^{* \overline{A(s)}}\left(O_{A}\right)\right\|_{\infty}\right) \\
\leqslant\left\|O_{A}\right\||A| \frac{J}{v} e^{v t} \nu_{\beta}^{-1}(s) .
\end{gathered}
$$

The second term is bounded using the rapid mixing condition:

$$
\left|\operatorname{tr} O_{A}\left(T_{t}^{\overline{A(s)}}\left(\rho_{\infty}\right)-\rho_{\infty}^{s}\right)\right| \leqslant\left\|O_{A}\right\| c|A|^{\delta} p(s) e^{-\gamma t} .
$$

By making the same choice of $t=t(s)$ as in lemma 6.2 , we get the desired bound. 
Corollary 6.5 (Approximate frustration-freeness). Under the same notation and assumptions of lemma 6.2. denote by $\rho_{\infty}$ the unique fixed point of $T_{t}$, and by $\rho$ a periodic point of $T_{t}^{A(s)}$. Then, we have the following bound:

$$
\sup _{\left\|O_{A}\right\|=1}\left|\operatorname{tr} O_{A}\left(\rho_{\infty}-\rho\right)\right| \leqslant 2\left\|O_{A}\right\|\left(\frac{J}{v}|A|+c|A|^{\delta}\right) \Delta_{0}(s) .
$$

Proof. By the triangle inequality and lemmas 6.2 and 6.4 , we have:

$$
\left.\mid \operatorname{tr} O_{A}\left(\rho_{\infty}-\rho\right)\right)|\leqslant| \operatorname{tr} O_{A}\left(\rho_{\infty}-\rho_{\infty}^{s}\right)|+| \operatorname{tr} O_{A}\left(\rho_{\infty}^{s}-\rho\right) \mid \leqslant
$$

$$
\leqslant 2\left\|O_{A}\right\|\left(\frac{J}{v}|A|+c|A|^{\delta}\right) \Delta_{0}(s)
$$

\subsection{Step 2: from global to local rapid mixing}

As a second step in the proof, we show that the assumptions on $\mathcal{L}$ imply local rapid mixing.

Proposition 6.6 (From global to local rapid mixing). Let $\mathcal{L}$ be a uniform family of Lindbladians with unique fixed point. Then, if condition 111 is satisfied, $\mathcal{L}$ satisfies local rapid mixing.

Proof. Let $O_{A}$ be an observable supported on $A$ with $\left\|O_{A}\right\|=1$. Denote by $s_{0}$ the minimum $s \geqslant 0$ such that $A(s)=\Lambda$. Fix $0 \leqslant s \leqslant s_{0}$, and let $B=A(s)$. Then, by the triangle inequality, we can bound the norm of $\left(T_{t}^{*}-T_{\infty}^{*}\right)$ as follows:

$$
\left\|\left(T_{t}^{*}-T_{\infty}^{*}\right) O_{A}\right\| \leqslant\left\|\left(T_{t}^{*}-T_{t}^{\bar{B} *}\right) O_{A}\right\|+\left\|\left(T_{t}^{\bar{B} *}-T_{\infty}^{\bar{B} *}\right) O_{A}\right\|+\left\|\left(T_{\infty}^{\bar{B} *}-T_{\infty}^{*}\right) O_{A}\right\| .
$$

We bound the first term on the right using lemmas 5.5 and 5.6 .

$$
\left\|\left(T_{t}^{*}-T_{t}^{\bar{B} *}\right) O_{A}\right\| \leqslant|A| \frac{J}{v}\left(e^{v t}-1-v t\right) e^{-\beta s} .
$$

The second term is bounded by the rapid mixing condition (11), setting $p(s)=(|A(s)| /|A|)^{\delta}$ :

$$
\left\|\left(T_{t}^{\bar{B} *}-T_{\infty}^{\bar{B} *}\right) O_{A}\right\| \leqslant \eta\left(T_{t}^{\bar{B}}\right) \leqslant c|A|^{\delta} p(s) e^{-\gamma t} .
$$

Finally, the third term is bounded by using lemma 6.4

$$
\left\|\left(T_{\infty}^{\bar{B} *}-T_{\infty}^{*}\right) O_{A}\right\|=\left|\operatorname{tr} O_{A}\left(\rho_{\infty}^{s}-\rho_{\infty}\right)\right| \leqslant\left(\frac{J}{v}|A|+c|A|^{\delta}\right) \Delta_{0}(s) .
$$

Substituting bounds (36), (37) and $(38)$ into equation 335$)$, we obtain, for $0 \leqslant s \leqslant s_{0}$ and for all $t \geqslant 0$ :

$$
\eta^{A}\left(T_{t}\right) \leqslant \frac{J}{v}|A| e^{v t} e^{-\beta s}+c|A|^{\delta} p(s) e^{-\gamma t}+\left(\frac{J}{v}|A|+c|A|^{\delta}\right) \Delta_{0}(s) .
$$

We want to show that we can choose $s=s(t) \in\left[0, s_{0}\right]$ in such a way that both $e^{v t} e^{-\beta s}$ and $e^{-t \gamma} p(s)$ are exponentially decaying in $t$. Choose $s:=s(t)=t(v+\gamma) / \beta$. Since $\Delta_{0}(s)=(|A(s)| /|A|)^{\delta v /(v+\gamma)} \nu_{\beta^{\prime}}^{-1}(s)$, denoting

$$
\bar{p}(t)=p \circ s(t)=p(t(v+\gamma) / \beta),
$$

we have that

$$
\Delta_{0}(s(t))=\bar{p}(t)^{v /(v+\gamma)} e^{-\gamma t} .
$$


Therefore, since $\bar{p}(t) \geqslant 1$,

$$
\begin{aligned}
\eta^{A}\left(T_{t}\right) \leqslant \frac{J}{v}|A| e^{-\gamma t}+c|A|^{\delta} \bar{p}(t) e^{-\gamma t}+\left(\frac{J}{v}|A|+c|A|^{\delta}\right) & \bar{p}(t)^{v /(v+\gamma)} e^{-\gamma t} \leqslant \\
& \leqslant 2\left(\frac{J}{v}|A|+c|A|^{\delta}\right) \bar{p}(t) e^{-\gamma t}, \quad \forall t \leqslant \frac{\beta}{v+\gamma} s_{0} .
\end{aligned}
$$

When $t \geqslant \beta /(v+\gamma) s_{0}$, we can simply bound $\eta^{A}\left(T_{t}\right)$ by $\eta\left(T_{t}\right)$ (see remark 5.8 , obtaining:

$$
\eta^{A}\left(T_{t}\right) \leqslant c|A|^{\delta} p\left(s_{0}\right) e^{-\gamma t} \leqslant c|A|^{\delta} \bar{p}(t) e^{-\gamma t}, \quad \forall t \geqslant \frac{\beta}{v+\gamma} s_{0}
$$

This completes the proof.

\subsection{Step 3: from local rapid mixing to stability}

We now prove that local rapid mixing alone implies stability. This is the last step in the proof of theorem 4.3 as we already proved in the previous sections that the condition of theorem 4.3 imply local rapid mixing. However, the following result also stands independently: if a system can be shown to satisfy local rapid mixing by other means, it will also be stable. Moreover, the same proof holds if we relax the assumption on prefactor $k(|A|)$ in equation 229 : a similar (but weaker) stability result will hold true as long as $|A|$ is independent of system size.

Theorem 6.7. Let $\mathcal{L}$ be a local Lindbladian satisfying local rapid mixing, and having a unique fixed point $\rho_{\infty}$ such that

$$
T_{\phi}^{*}\left(O_{A}\right)=T_{\infty}^{*}\left(O_{A}\right)=\operatorname{tr}\left(O_{A} \rho_{\infty}\right) \mathbb{1} .
$$

Then, using the notation of theorem 4.3, for all observables $O_{A}$ supported on $A \subset \Lambda$ we have that

$$
\left\|T_{t}^{*}\left(O_{A}\right)-S_{t}^{*}\left(O_{A}\right)\right\| \leqslant c(|A|)\left\|O_{A}\right\|\left(\varepsilon+|\Lambda| \nu_{\eta}^{-1}\left(d_{A}\right)\right),
$$

where $d_{A}=\operatorname{dist}\left(A, \Lambda^{c}\right) ; \eta$ is positive and independent of $\Lambda ; \nu_{\eta}^{-1}(d) \leqslant(1+d)^{-D-1} ; c(|A|)$ is independent of $\Lambda$ and $t$, and is bounded by a polynomial in $|A|$.

Proof. Let $O_{0}(t)=T_{t}^{*}\left(O_{A}\right)$ and $O_{1}(t)=S_{t}^{*}\left(O_{A}\right)$ and write the difference $O_{0}-O_{1}$ using the integral representation from equation 21):

$$
O_{0}(t)-O_{1}(t)=\int_{0}^{t} S_{t-s}^{*} E^{*} T_{s}^{*}\left(O_{A}\right) \mathrm{d} s .
$$

The triangle inequality implies:

$$
\left\|O_{0}(t)-O_{1}(t)\right\| \leqslant \sum_{u} \sum_{r} \int_{0}^{t}\left\|E_{u, r}^{*} O_{0}(s)\right\| \mathrm{d} s+\sum_{d} \int_{0}^{t}\left\|E_{d}^{*} O_{0}(s)\right\| \mathrm{d} s,
$$

where we used the fact that $S_{t}$ is a contraction.

Fix a $K \in\left\{E_{u, r}\right\}_{u, r} \cup\left\{E_{d}\right\}_{d}$, and let $\delta=\operatorname{dist}\left(A\right.$, supp $K$ ). We can split the integral at a time $t_{0}$ (to be fixed later, depending on $\delta$ ). We bound the first part of the integral with Lieb-Robinson bounds:

$$
\int_{0}^{t_{0}}\left\|K^{*} O_{0}(s)\right\| \mathrm{d} s \leqslant\|K\|_{1 \rightarrow 1, c b}\left\|O_{A}\right\||A| \frac{e^{v t_{0}}-v t_{0}-1}{v \nu_{\mu}(\delta)} .
$$


Now pick $t_{0}=t_{0}(\delta)$ such that

$$
\nu_{\mu}^{-1}(\delta) \frac{e^{v t_{0}}-v t_{0}-1}{v} \leqslant \nu_{\mu / 2}^{-1}(\delta) .
$$

We can choose $t_{0}(\delta)=\frac{\mu}{2} \frac{\log v}{v} \delta=O(\delta)$, for exponentially decaying (or faster) $\nu_{\mu}^{-1}(\delta)$.

If $t \leqslant t_{0}(\delta)$, then we have bounded the entire integral, and we are done. Otherwise, we treat the second part of the integral as follows:

$$
\begin{aligned}
& \int_{t_{0}(\delta)}^{t}\left\|K^{*} O_{0}(s)\right\| \mathrm{d} s=\int_{t_{0}(\delta)}^{t}\left\|K^{*}\left(O_{0}(s)-T_{\infty}^{*}\left(O_{A}\right)\right)\right\| \mathrm{d} s \\
& \leqslant\|K\|_{1 \rightarrow 1, c b}\left\|O_{A}\right\| \int_{t_{0}(\delta)}^{\infty} \eta^{A}\left(T_{s}\right) \mathrm{d} s \leqslant\|K\|_{1 \rightarrow 1, c b}\left\|O_{A}\right\| q(|A|) \int_{t_{0}(\delta)}^{\infty} e^{-\gamma s} \mathrm{~d} s \\
& =\|K\|_{1 \rightarrow 1, c b}\left\|O_{A}\right\| k(|A|) \frac{1}{\gamma} e^{-\gamma t_{0}(\delta)}
\end{aligned}
$$

where we used $K^{*} T_{\infty}^{*}\left(O_{A}\right)=K^{*}\left(\operatorname{tr}\left(\rho_{\infty} O_{A}\right) \mathbb{1}\right)=\operatorname{tr}\left(\rho_{\infty} O_{A}\right) K^{*}(\mathbb{1})=0$, together with the local rapid mixing condition.

Since $t_{0}(\delta)$ is linear in $\delta$, we have that:

$$
h(\delta):=e^{-\frac{\mu \delta}{2}}+\frac{1}{\gamma} e^{-\gamma t_{0}(\delta)}
$$

is exponentially decaying in $\delta$.

Putting the different bounds together, we obtain:

$$
\int_{0}^{t}\left\|K^{*} O_{0}(s)\right\| \mathrm{d} s \leqslant\|K\|_{1 \rightarrow 1, c b}\left\|O_{A}\right\| k_{1}(|A|) h(\delta),
$$

where $k_{1}(|A|)=\max (k(|A|),|A|)$.

Returning to the sum, we have proven that:

$$
\begin{aligned}
\left\|O_{0}(t)-O_{1}(t)\right\| \leqslant \varepsilon k_{1}(|A|)\left\|O_{A}\right\|[\underbrace{\sum_{u} \sum_{r} e(r) h\left(\operatorname{dist}\left(A, b_{r}(u)\right)\right)}_{I_{1}(A ; e, h)} & \\
& +\underbrace{\sum_{d}\left|\partial_{d} \Lambda\right| e(d) h\left(\operatorname{dist}\left(A, \partial_{d} \Lambda\right)\right)}_{I_{2}(A ; e, h)}] .
\end{aligned}
$$

It suffices to show that $I_{1}$ and $I_{2}$ are finite (and independent of system size), and that $I_{2}$ decays exponentially in $\operatorname{dist}\left(A, \Lambda^{c}\right)$. Let us decompose the $I_{1}$ as follows

$$
\begin{aligned}
& I_{1}(A ; e, h)=\sum_{u} \sum_{r} e(r) h\left(\operatorname{dist}\left(A, b_{r}(u)\right)\right) \\
& =\sum_{\operatorname{dist}(u, A)=0} \sum_{r} e(r) h(0)+\sum_{d>0} \sum_{\operatorname{dist}(u, A)=d}\left(\sum_{r=0}^{d} e(r) h(d-r)+\sum_{r=d+1}^{\infty} e(r) h(0)\right) \\
& =h(0)|A| \sum_{r} e(r)+\sum_{d>0} q(d)\left(\sum_{r=0}^{d} e(r) h(d-r)+h(0) \sum_{r=d+1}^{\infty} e(r)\right),
\end{aligned}
$$


where $q(d)=|\{u: \operatorname{dist}(u, A)=d\}|$ grows polynomially in $d$.

The first term is clearly bounded, since $e(r)$ is summable. Since $e$ and $h$ are both exponentially decaying functions, their discrete convolution $e \star h(d)=\sum_{r=0}^{d} e(r) h(d-r)$ is also exponentially decaying, and consequently summable against any polynomial. The same holds for $\sum_{r>d} e(r)$. This proves that the second term is also bounded.

On the other hand, we have that

$$
I_{2}(A ; e, h)=\sum_{d}\left|\partial_{d} \Lambda\right| e(d) h\left(\operatorname{dist}\left(A, \partial_{d} \Lambda\right)\right) \leqslant|\Lambda|\left(e \star h\left(d_{A}\right)+\sum_{d \geqslant d_{A}} e(d)\right),
$$

where $d_{A}=\operatorname{dist}\left(A, \Lambda^{c}\right)$. We have just proven that $e \star h\left(d_{A}\right)$ and $\sum_{d \geqslant d_{A}} e(d)$ are exponentially decaying. This implies that there exists a positive $\eta$ such that $\nu_{\eta}^{-1}\left(d_{A}\right)$ upper bounds both. Denoting $c(|A|)=$ $k_{1}(|A|) I_{1}(A ; e, h)$, we have the desired bound.

\subsection{Power-law decay}

As we stated before, the results and proofs presented above still hold when $\mathcal{L}$ has quasi-local or power-law interactions. In the latter case, this is only true when certain conditions are met on the decay of $\mathcal{L}$. In what follows, we highlight the changes one needs to make in the case of power-law decay, in order for the main stability results to hold.

Definition 6.8 (Compatibility condition). Let $\mathcal{L}$ be a local Lindbladian, and suppose it satisfies (LR-2 and rapid mixing $\operatorname{RM}(\gamma, \delta)$. Let $\mu$ and $v$ be the Lieb-Robinson constants for $\mathcal{L}$ defined in assumption 5.1 and $\beta$ the constant defined in lemma 5.5 Then we say that $\mathcal{L}$ satisfies the weak compatibility condition for stability, if the following inequality is satisfied.

$$
\beta \gamma-\delta D v>0
$$

we say that $\mathcal{L}$ satisfies the strong compatibility condition for stability if

$$
\mu \frac{\beta \gamma-\delta D v}{\beta(\gamma+v)}>D+2
$$

Moreover, if the perturbation $E$, defined in theorem 4.3 is decaying polynomially and not exponentially, it must satisfy

$$
\sum_{n} n^{D} \sum_{r>n} e(r)<\infty
$$

for the theorem to hold.

Remark 6.9. Clearly, the strong version of the compatibility condition implies the weak one. If $\mathcal{L}$ has quasilocal interactions, then the (polynomial) decay rate $\alpha$ of the interactions can be chosen to be larger than any fixed value. Consequently, since $\beta$ and $\mu$ can be taken to be linear in $\alpha$, quasi-local Lindbladians $\mathcal{L}$ satisfy the strong compatibility condition $\mathrm{CC}-2$.

Under the weak compatibility condition, all the results presented in sections 6.1 and 6.2 still hold true, while under the strong compatibility condition also the results presented in 6.3 are still valid, and in particular our main result, theorem 6.7

We will now show this in the cases in which we made explicit use of condition (LR-1), and give the needed modifications to the proofs of lemma 6.2 proposition 6.6 and theorem 6.7 in order to make them valid for power-law decaying interactions.

From now on, we proceed under the working hypothesis that $\mathcal{L}$ satisfies $(\mathrm{LR}-2$ and that the above compatibility conditions are satisfied. 
Modifications in the proof of lemma 6.2. The argument below follows closely the proof of the original lemma, but now one must check that $\Delta_{0}(s)$ is still decaying. Recall the definition of $\Delta_{0}(s)$ from the original proof of lemma 6.2 ,

$$
\Delta_{0}(s)=(|A(s)| /|A|)^{\delta v /(v+\gamma)} \nu_{\beta^{\prime}}^{-1}(s), \quad \beta^{\prime}=\beta \gamma /(v+\gamma) .
$$

Since $(|A(s)| /|A|)^{\delta v /(v+\gamma)}$ grows as $(1+s)^{\delta D v /(v+\gamma)}$, we have:

$$
\Delta_{0}(s) \sim(1+s)^{-\gamma^{\prime}},
$$

where $\gamma^{\prime}=\frac{\beta \gamma-\delta D v}{v+\gamma}$ is positive because of $\mathrm{CC}-1$.

Modifications in the proof of proposition 6.6. Keeping the notation introduced in the original proof of this proposition, we have already shown that, for each $0 \leqslant s \leqslant s_{0}$ :

$$
\eta^{A}\left(T_{t}\right) \leqslant \frac{J}{v}|A| e^{v t} \nu_{\beta}^{-1}(s)+c|A|^{\delta} p(s) e^{-\gamma t}+\left(\frac{J}{v}|A|+c|A|^{\delta}\right) \Delta_{0}(s) .
$$

At this point, we can no longer choose $s=s(t)$ to scale linearly in $t$, since the decay $\nu_{\beta}^{-1}(s)$ is polynomial in $s$ and the prefactor $e^{v t}$ would render the bound trivial. Still, we may choose $s=s(t) \in\left[0, s_{0}\right]$ in such a way that the r.h.s. above is exponentially decaying in $t$.

Fix $k>0$ (to be determined later), and consider:

$$
s(t)=e^{k t}-1,
$$

in such a way that for $t \leqslant \log \left(1+s_{0}\right) / k$, we have:

$$
\bar{p}(t)=p \circ s(t)=\left(\left|A\left(e^{k t}-1\right)\right| /|A|\right)^{\delta} \sim e^{k D \delta t} .
$$

Then, the r.h.s. of the desired bound for $\eta^{A}\left(T_{t}\right)$ contains the following exponentials:

$$
e^{v t} \nu_{\beta}^{-1}(s)=e^{-(\beta k-v) t} ; \quad p(s) e^{-\gamma t} \sim e^{-(\gamma-k D \delta) t},
$$

and

$$
\Delta_{0}(s) \sim(1+s)^{-\gamma^{\prime}}=e^{-k \gamma^{\prime} t}
$$

where

$$
\gamma^{\prime}=\frac{\beta \gamma-\delta D v}{v+\gamma}
$$

is defined in the modified proof of lemma 6.2. We want to show that we can choose $k$ in such a way that all the exponential functions appearing above are decaying, i.e. each exponent is negative for $t>0$. (CC-1) implies that $\Delta_{0}(s)$ is decaying for all $k>0$. Let

$$
k^{\prime}=\frac{v+\gamma}{\beta+\delta D},
$$

such that $\beta k^{\prime}-v=\gamma-k^{\prime} D \delta=k^{\prime} \gamma^{\prime}$, making all of the above exponents equal to $-(\beta \gamma-\delta D v) /(\beta+\delta D)$ and negative (due to (CC-1)), as desired.

When $t \geqslant \log \left(1+s_{0}\right) / k^{\prime}$, as in the proof for exponentially decaying interactions, we bound $\eta^{A}\left(T_{t}\right)$ by $\eta\left(T_{t}\right)$ (see remark 5.8), thus obtaining:

$$
\eta^{A}\left(T_{t}\right) \leqslant c|A|^{\delta} p\left(s_{0}\right) e^{-\gamma t} \leqslant c|A|^{\delta} \bar{p}(t) e^{-\gamma t} \sim c|A|^{\delta} e^{-k^{\prime} \gamma^{\prime} t} .
$$


Modifications in the proof of theorem 6.7. Following the same steps as in the original proof, but now using the constants for the local rapid mixing obtained in the modified proof of proposition 6.6. we have that, for each $0 \leqslant t_{0} \leqslant t$ :

$$
\int_{0}^{t}\left\|K^{*} O_{0}(s)\right\| \mathrm{d} s \quad \leqslant \quad\|K\|_{1 \rightarrow 1, c b}\left\|O_{A}\right\|\left(|A| \frac{1}{v} e^{v t_{0}} \nu_{\mu}^{-1}(d)+k(|A|) e^{-t_{0} \frac{\beta \gamma-\delta D v}{\beta+\delta D}}\right),
$$

where $d=\operatorname{dist}(A, \operatorname{supp} K)$.

Let us define $t_{0}(d)=k \log (1+d)$ for some positive $k$ (to be determined later), and denote $h(d)=$ $\nu_{v k-\mu}(d)+\nu_{-k \frac{\beta \gamma-\delta D v}{\beta+\delta D}}(d)$, such that

$$
\int_{0}^{t}\left\|K O_{0}(s)\right\| \mathrm{d} s \leqslant\|K\|_{1 \rightarrow 1, c b}\left\|O_{A}\right\| k_{1}(|A|) h(d)
$$

where $k_{1}(|A|)=\max (k(|A|),|A| / v)$. Then we have that $h$ has a maximum decay rate of

$$
\mu^{\prime}=\sup _{k \geqslant 0} \min \left(\mu-v k, k \frac{\beta \gamma-\delta D v}{\beta+\delta D}\right) .
$$

The optimal choice of $k$ is $k=\frac{\mu}{\beta} \frac{\beta+\delta D}{v+\gamma}$, in such a way that $\mu^{\prime}=\frac{\mu}{\beta} \frac{\beta \gamma-\delta D v}{v+\gamma} . \mu^{\prime}$ is positive because of condition (CC-1).

Recalling the following definitions from the original proof of theorem 6.7 .

$$
\begin{array}{ll}
q(d)=|\{u: \operatorname{dist}(u, A)=d\}|, & l(d)=\left|\partial_{d} \Lambda\right| e(d), \\
x \star y(d)=\sum_{r=0}^{d} x(r) y(d-r), & d_{A}=\operatorname{dist}\left(A, \Lambda^{c}\right),
\end{array}
$$

we need to show that

$$
I_{1}(A ; e, h)=h(0)|A| \sum_{r} e(r)+\sum_{d>0} q(d)\left(e \star h(d)+h(0) \sum_{r>d} e(r)\right) e \star h(d)
$$

is finite, and that

$$
I_{2}(A ; e, h)=l \star h\left(d_{A}\right)+\sum_{d \geqslant d_{A}} l(d) \leqslant \nu_{\eta}^{-1}\left(d_{A}\right)
$$

for some positive $\eta$. Notice that

$$
I_{1}(A ; e, h) \leqslant(1+|A|) h(0) \sum_{d} q(d) \sum_{r>d} e(r)+\sum_{d} q(d) e \star h(d) .
$$

Since $q(d)$ grows as $(1+d)^{D}, \sum_{d} q(d) \sum_{r \geqslant d} e(r)$ is finite if $\sum_{n} n^{D} \sum_{r>n} e(r)<\infty$, which is condition (CC-e).

On the other hand, $e \star h$ decays as the slowest of the two functions. Since we have already assumed that $\sum_{d} q(d) \sum_{r>d} e(r)$ is finite, we only need to satisfy that $\sum_{d} q(d) \sum_{r>d} h(r)$ is finite. For this to happen, it suffices that $\mu^{\prime}>D+2$, which is condition (CC-2).

In order to bound $I_{2}(A ; e, h)$, note that $l(d) \leqslant|\Lambda| e(d)$, and therefore

$$
I_{2}(A ; e, h) \leqslant|\Lambda|\left(e \star h\left(d_{A}\right)+\sum_{d \geqslant d_{A}} e(d)\right) .
$$

We have already proven that conditions $\mathrm{CC}-2$ and $\mathrm{CC}-\mathrm{e}$ imply that the r.h.s. of the latter bound is decaying polynomially in $d_{A}$ at least as fast as $\nu_{D+1}^{-1}\left(d_{A}\right)$.

This concludes the proof. 


\subsection{Relaxing rapid mixing}

In this section, we will show that, in the case of exponentially decaying interactions (LR-1), the proof of theorem 4.3 still holds if

$$
\sum_{n} n^{D} \sum_{d>n} \int_{d}^{\infty}\left(1+\frac{v}{\beta} s-\frac{1}{\beta} \log \gamma(s)\right)^{\delta D} \gamma(s) \mathrm{d} s<\infty .
$$

We will directly prove proposition 6.6 without the intermediate step of a lemma like 6.2 Nonetheless, results of that kind can be proven using exactly the same arguments that we will use in the following proof.

Proof of proposition 6.6. Using the same notation as in the original proof, we have that for $0 \leqslant s \leqslant s_{0}$ :

$$
\left\|\left(T_{t}^{*}-T_{\infty}^{*}\right) O_{A}\right\| \leqslant\left\|\left(T_{t}^{*}-T_{t}^{\bar{B} *}\right) O_{A}\right\|+\left\|\left(T_{t}^{\bar{B} *}-T_{\infty}^{\bar{B} *}\right) O_{A}\right\|+\left\|\left(T_{\infty}^{\bar{B} *}-T_{\infty}^{*}\right) O_{A}\right\| .
$$

We will bound the first two terms as in the original proof (using lemma 5.5 lemma 5.6 and equation (15) while we rewrite the third term as in the proof of lemma 6.2

$$
\begin{aligned}
\left\|\left(T_{\infty}^{\bar{B} *}-T_{\infty}^{*}\right) O_{A}\right\|= & \left|\operatorname{tr} O_{A}\left(\rho_{\infty}^{s}-\rho_{\infty}\right)\right| \leqslant \\
& \left|\operatorname{tr} O_{A}\left(\rho_{\infty}-T_{t}^{\overline{A(s)}}\left(\rho_{\infty}\right)\right)\right|+\left|\operatorname{tr} O_{A}\left(T_{t}^{\overline{A(s)}}\left(\rho_{\infty}\right)-\rho_{\infty}^{s}\right)\right|= \\
& \left|\operatorname{tr} O_{A}\left(T_{t}\left(\rho_{\infty}\right)-T_{t}^{\overline{A(s)}}\left(\rho_{\infty}\right)\right)\right|+\left\|O_{A}\right\|\left\|T_{t}^{\overline{A(s)}}\left(\rho_{\infty}\right)-\rho_{\infty}^{s}\right\| \|_{1} \leqslant \\
& \left\|\left(T_{t}^{*}-T_{t}^{\bar{B} *}\right) O_{A}\right\|+\eta\left(T_{t}^{\bar{B}}\right) .
\end{aligned}
$$

Thus we have that

$$
\left\|\left(T_{t}^{*}-T_{\infty}^{*}\right) O_{A}\right\| \leqslant 2 \frac{J}{v}|A| e^{v t} \nu_{\beta}^{-1}(s)+2 c|A|^{\delta} p(s) \gamma(t),
$$

where $p(s)=\left(\mid(A(s)|/| A \mid)^{\delta} \sim(1+s)^{\delta D}\right.$.

We have claimed that the result only holds in the case of exponentially decaying or faster decay of interaction. Suppose $\nu_{\beta}(s)=(1+s)^{\beta}$ (i.e., if $\mathcal{L}$ satisfies $(\overline{\text { LR-2 }})$ ). Defining $s=s(t)$ as

$$
s(t)=e^{\frac{v}{\beta+\delta D} t} \gamma(t)^{-\frac{1}{\beta+\delta D}}-1,
$$

then it holds that

$$
\frac{e^{v t}}{\gamma(t)}=p(s(t)) \nu_{\beta}(s(t)) \quad \forall t \leqslant t_{0},
$$

where $t_{0}$ is such that $s\left(t_{0}\right)=s_{0}$. Thus

$$
\delta_{0}(t):=e^{v t} \nu_{\beta}^{-1}(s)=e^{\frac{\delta D}{\beta+\delta D} v t} \gamma(t)^{\frac{\beta}{\beta+\delta D}} .
$$

We have that this last function is decaying in $t$ if

$$
\gamma(t)<e^{-(v \delta D / \beta) t}
$$

This forces $\gamma(t)$ to be exponentially decaying, and thus there is no possible relaxation of the rapid mixing condition.

On the other hand, if $\nu_{\beta}(s)=e^{\beta s}$ (i.e., if $\mathcal{L}$ satisfies (LR-1)), we define

$$
s(t)=\frac{v}{\beta} t-\frac{1}{\beta} \log \gamma(t),
$$


such that $e^{v t} \nu_{\beta}^{-1}(s)=\gamma(t)$ and

$$
\bar{p}(t)=p \circ s(t) \sim\left(1+\frac{v}{\beta} t-\frac{1}{\beta} \log \gamma(t)\right)^{\delta D}
$$

grows polynomially.

In this case, we have proved that

$$
\left\|\left(T_{t}^{*}-T_{\infty}^{*}\right) O_{A}\right\| \leqslant 2\left(\frac{J}{v}|A|+c|A|^{\delta}\right) \bar{p}(t) \gamma(t),
$$

and this concludes the proof since equation (41) implies that $\bar{p}(t) \gamma(t)$ is decaying in $t$.

Proof of theorem 6.7. Following the same steps as in the original proof, we have that for any $K \in\left\{E_{u, r}\right\}_{u, r} \cup$ $\left\{E_{d}\right\}_{d}$, and let $\delta=\operatorname{dist}(A, \operatorname{supp} K)$

$$
\int_{0}^{t}\left\|K^{*} O_{0}(s)\right\| \mathrm{d} s \leqslant\|K\|_{1 \rightarrow 1, c b}\left\|O_{A}\right\| k_{1}(|A|) h(\delta) .
$$

where $h(\delta)$ is now

$$
h(\delta)=e^{-\mu \delta / 2}+\int_{\frac{\mu}{2} \frac{\log v}{v} \delta}^{\infty} \bar{p}(s) \gamma(s) \mathrm{d} s .
$$

We want to show that $h(\delta)$ is decaying fast enough for the r.h.s. of equation 40 to be summable. This is the case (see the proof of theorem 6.7 in section 6.4 if equation 441) holds.

\section{Glauber dynamics}

\subsection{Quantum embedding of Glauber dynamics}

As an example of a non-trivial dynamics for which we can now prove stability using our results, we turn to one of the most studied dynamics in classical statistical mechanics: Glauber dynamics, a Markov process that samples thermal states of local (classical) Hamiltonians on lattices. Apart from being an interesting model in itself, it has important applications in Monte-Carlo Markov chain algorithms for numerical manybody physics [41]. Determining whether Glauber dynamics is stable against noise or errors is therefore an important question and, as far as we are aware, still open (with partial results obtained under the assumption of attractiveness [27]).

In this section, we present a natural embedding of Glauber dynamics into the Linbdlabian setting, showing how this embedded dynamics inherits properties from the classical Markov chain 8 We will then apply the results of section 4 to prove, in the appropriate regime, stability of Glauber dynamics.

We will consider a lattice spin system over $\Gamma=\mathbb{Z}^{D}$ or $\Gamma=(\mathbb{Z} / L \mathbb{Z})^{D}$, with (classical) configuration space of a single spin a finite set $S$. For simplicity, we will consider the case $S=\{+1,-1\}$. For each $\Lambda \subset \Gamma$, we will denote by $\Omega_{\Lambda}$ the space of configurations over $\Lambda$, namely $S^{\Lambda} . \Lambda^{c}$ will denote the complementary of $\Lambda$ in $\Gamma$, namely $\Gamma \backslash \Lambda$.

Definition 7.1. A finite range, translationally-invariant potential $\left\{J_{A}\right\}_{A \subset \Gamma}$ is a family of real functions indexed by the non empty finite subsets of $\Gamma$ satisfying the following properties:

1. $J_{A}: \Omega_{A} \rightarrow \mathbb{R}$.

\footnotetext{
8 A similar construction was proposed in 4 .
} 
2. For all $A \subset \Gamma$ and all $x \in \Gamma$ :

$$
J_{A}(\sigma)=J_{A+x}(\eta) \quad \text { if } \quad \sigma(y+x)=\eta(y) \quad \forall y \in A .
$$

3. There exists a positive $r>0$ such that $J_{A}=0$ if $\operatorname{diam} A>r$, called the range of interaction.

Given a finite-range, translationally-invariant potential, we can define a Hamiltonian for each finite lattice $\Lambda \subset \Gamma$ and each boundary condition $\tau \in \Omega_{\Lambda^{c}}$ by

$$
H_{\Lambda}^{\tau}(\sigma)=-\sum_{A \cap \Lambda \neq 0} J_{A}(\sigma \times \tau) \quad \forall \sigma \in \Omega_{\Lambda}
$$

where $\sigma \times \tau$ is the configuration that agrees with $\sigma$ over $\Lambda$ and with $\tau$ over $\Lambda^{c}$. For each such Hamiltonian, we define the Gibbs state state as

$$
\mu_{\Lambda}^{\tau}(\sigma)=\left(Z_{\Lambda}^{\tau}\right)^{-1} \exp \left(-H_{\Lambda}^{\tau}(\sigma)\right)
$$

where $Z_{\Lambda}^{\tau}$ is a normalizing constant 9 The convex hull of the set of Gibbs states over $\Lambda$ will be denoted by $\mathcal{G}(\Lambda)$ :

$$
\mathcal{G}(\Lambda)=\operatorname{conv}\left\{\mu_{\Lambda}^{\tau} \mid \tau \in \Omega_{\Lambda^{c}}\right\}
$$

Definition 7.2. The Glauber dynamics for a potential $J$ is the Markov process on $\Omega_{\Lambda}$ with the following generator:

$$
\left(Q_{\Lambda} f\right)(\sigma)=\sum_{x \in \Lambda} c_{J}(x, \sigma) \nabla_{x} f(\sigma)
$$

where $\nabla_{x} f(\sigma)$ if defined as $f\left(\sigma^{x}\right)-f(\sigma)$, and $\sigma^{x}$ is the configuration obtained by flipping the spin at position $x$ :

$$
\sigma^{x}(y)= \begin{cases}\sigma(y) & \text { if } x \neq y \\ -\sigma(x) & \text { if } x=y\end{cases}
$$

The numbers $c_{J}(x, \sigma)$ are called transition rates and must satisfy the following assumptions:

1. Positivity and boundedness: There exist positive constants $c_{m}$ and $c_{M}$ such that:

$$
0<c_{m} \leqslant c_{J}(x, \sigma) \leqslant c_{M}<\infty \quad \forall x, \sigma .
$$

2. Finite range: $c_{J}(x, \cdot)$ depends only on spin values in $b_{r}(x)$.

3. Translational invariance: for all $k \in \Gamma$,

$$
c_{J}\left(x, \sigma^{\prime}\right)=c_{J}(x+k, \sigma) \quad \text { if } \quad \sigma^{\prime}(y)=\sigma(y+k) \quad \forall y .
$$

4. Detailed balance: for all $x \in \Gamma$ and all $\sigma$

$$
\exp \left(-\sum_{A \ni x} J_{A}(\sigma)\right) c_{J}(x, \sigma)=c_{J}\left(x, \sigma^{x}\right) \exp \left(-\sum_{A \ni x} J_{A}\left(\sigma^{x}\right)\right) .
$$

These assumptions are sufficient to ensure that $Q_{\Lambda}$ generates a Markov process which has the Gibbs states over $\Lambda$ as stationary points.

$$
J \text {. }
$$

${ }^{9}$ Following 47 , in our notation we have incorporated the usual inverse temperature parameter $\beta$ directly into the potential 
Definition 7.3. A quantum embedding of the classical Glauber dynamics for a potential $J$ is generated by the following Lindblad operators

$$
\begin{gathered}
L_{x, \eta}=\sqrt{c_{J}(x, \eta)}\left|\eta^{x}\right\rangle\langle\eta| \otimes \mathbb{1}, \quad \forall x \in \Lambda, \forall \eta \in \Omega_{b_{x}(r)} ; \\
\mathcal{L}_{x, \eta}(\rho)=L_{x, \eta} \rho L_{x, \eta}^{*}-\frac{1}{2}\left\{\rho, c_{J}(x, \eta)|\eta\rangle\langle\eta|\right\} ; \\
\mathcal{L}_{\Lambda}(\rho)=\sum_{x \in \Lambda} \sum_{\eta} L_{x, \eta} \rho L_{x, \eta}^{*}-\frac{1}{2}\{\rho, K\}, \quad K=\sum_{\sigma}\left(\sum_{x} c_{J}(x, \sigma)\right)|\sigma\rangle\langle\sigma| ;
\end{gathered}
$$

plus a dephasing channel acting independently and uniformly on all sites $x \in \Lambda$ :

$$
D_{x, 0}=\sqrt{\gamma}|0\rangle\left\langle 0\left|, \quad D_{x, 1}=\sqrt{\gamma}\right| 1\right\rangle\left\langle 1\left|, \quad \mathcal{D}(\rho)=\sum_{x \in \Lambda} \sum_{i=0,1} D_{x, i} \rho D_{x, i}^{*}-\right| \Lambda\right| \gamma \rho .
$$

$\mathcal{L}_{\Lambda}$ satisfies translational invariance because the transition rates $c_{J}$ do, and it easy to see that this family of Lindbladians is uniform.

Remark 7.4. Take $|\alpha\rangle\langle\beta|$ an element of the computational basis, and let $d(\alpha, \beta)$ be the Hamming distance between $\alpha$ and $\beta$. Then it holds that

$$
\mathcal{D}(|\alpha\rangle\langle\beta|)=-\gamma d(\alpha, \beta)|\alpha\rangle\langle\beta| .
$$

In other words, $\mathcal{D}$ is a Schur multiplier in the computational basis, represented by $(-\gamma d(\alpha, \beta))_{\alpha, \beta}$.

On the other hand, we have that for all $x$ :

$$
\sum_{\eta \in \Omega_{b_{x}(r)}} \mathcal{L}_{x, \eta}(|\alpha\rangle\langle\beta|)= \begin{cases}c_{J}(x, \alpha)\left(\left|\alpha^{x}\right\rangle\left\langle\beta^{x}|-| \alpha\right\rangle\langle\beta|\right) & \text { if }\left.\alpha\right|_{b_{x}(r)}=\left.\beta\right|_{b_{x}(r)}, \\ -\frac{1}{2}\left(c_{J}(x, \alpha)+c_{J}(x, \beta)\right)|\alpha\rangle\langle\beta| & \text { otherwise. }\end{cases}
$$

Since $d\left(\alpha^{x}, \beta^{x}\right)=d(\alpha, \beta),\left[\mathcal{D}, \sum_{\eta} \mathcal{L}_{x, \eta}\right]=0$ for all $x \in \Lambda$, and in particular $\mathcal{D}$ and $\mathcal{L}_{\Lambda}$ commute.

This quantum dissipative system inherits various properties from its classical counterpart.

Definition 7.5. Let $\mu$ be a full-rank positive state. Denote by

$$
\Gamma_{\mu}(\rho)=\mu^{\frac{1}{2}} \rho \mu^{\frac{1}{2}} .
$$

We say that $\mathcal{L}$ is in detailed balance [37, 44, 45, 60, with respect to $\mu$ if $\Gamma_{\mu} \circ \mathcal{L}=\mathcal{L}^{*} \circ \Gamma_{\mu}$.

Proposition 7.6. Let $\mu_{\Lambda}^{\tau}$ be a Gibbs state over $\Lambda$. Then $\mathcal{L}_{\Lambda}$ and $\mathcal{D}$ are in detailed balance with respect to $\mu_{\Lambda}^{\tau}$.

Proof. Note that $\Gamma_{\mu_{\Lambda}^{\tau}}$ is a Schur multiplier in the computational basis:

$$
\Gamma_{\mu_{\Lambda}^{\tau}}\left(\left|\eta_{1}\right\rangle\left\langle\eta_{2}\right|\right)=\mu_{\Lambda}^{\tau}\left(\eta_{1}\right)^{\frac{1}{2}} \mu_{\Lambda}^{\tau}\left(\eta_{2}\right)^{\frac{1}{2}}\left|\eta_{1}\right\rangle\left\langle\eta_{2}\right| .
$$

From the detailed balance condition for the transition rates $c_{J}(x, \sigma)$, it follows that for all $x \in \Lambda$, denoting $\mathcal{L}_{x}=\sum_{\eta \in \Omega_{b_{x}(r)}} \mathcal{L}_{x, \eta}$

$$
\begin{aligned}
\Gamma_{\mu_{\Lambda}^{\tau}} & \circ \mathcal{L}_{x} \circ \Gamma_{\mu_{\Lambda}^{\tau}}^{-1}\left(\left|\eta_{1}\right\rangle\left\langle\eta_{2}\right|\right) \\
& =\delta_{\eta_{1}, \eta_{2}}^{x}\left(c_{J}\left(x, \eta_{1}\right) \frac{\mu_{\Lambda}^{\tau}\left(\eta_{1}^{x}\right)}{\mu_{\Lambda}^{\tau}\left(\eta_{1}\right)}\right)\left|\eta_{1}^{x}\right\rangle\left\langle\eta_{2}^{x}\left|-\frac{c_{J}\left(x, \eta_{1}\right)+c_{J}\left(x, \eta_{2}\right)}{2}\right| \eta_{1}\right\rangle\left\langle\eta_{2}\right| \\
& =\delta_{\eta_{1}, \eta_{2}}^{x} c_{J}\left(x, \eta_{1}^{x}\right)\left|\eta_{1}^{x}\right\rangle\left\langle\eta_{2}^{x}\left|-\frac{c_{J}\left(x, \eta_{1}\right)+c_{J}\left(x, \eta_{2}\right)}{2}\right| \eta_{1}\right\rangle\left\langle\eta_{2}\right| \\
& \left.=\mathcal{L}_{x}^{*}\left(\left|\eta_{1}\right\rangle\left\langle\eta_{2}\right|\right)\right),
\end{aligned}
$$


where

$$
\delta_{\eta_{1}, \eta_{2}}^{x}= \begin{cases}1 & \text { if }\left.\eta_{1}\right|_{b_{x}(r)}=\left.\eta_{2}\right|_{b_{x}(r)} \\ 0 & \text { otherwise. }\end{cases}
$$

To prove detailed balance for $\mathcal{D}$, note that Schur multipliers commute, thus $\left[\mathcal{D}, \Gamma_{\mu}\right]=0$. This, together with the fact that $\mathcal{D}^{*}=\mathcal{D}$, implies that $\mathcal{D}$ is in detailed balance w.r.t. $\mu_{\Lambda}^{\tau}$.

The above proposition implies that Gibbs states are stationary states for the quantum Glauber dynamics. Let us prove that there are no other fixed points apart from the classical ones (i.e. states that are diagonal in the computational basis). Clearly, $\mathcal{D}$ has all classical states as stationary points. We just have to check $\mathcal{L}_{\Lambda}$.

Proposition 7.7. The set of fixed points of $\mathcal{L}_{\Lambda}$ is equal to $\mathcal{G}(\Lambda)$, the set of Gibbs states over $\Lambda$.

Proof. Let $\rho$ be a fixed point of $\mathcal{L}_{\Lambda}$. We want to prove that $\rho$ is diagonal, i.e. that it is of the form

$$
\rho=\sum_{\sigma} p_{\sigma}|\sigma\rangle\langle\sigma|
$$

Consider a non-diagonal element $|\alpha\rangle\langle\beta|$, and suppose $\alpha(x) \neq \beta(x)$ for some $x \in \Lambda$. Then, from equation 45, we have that for all $y \in b_{x}(r)$,

$$
\mathcal{L}_{y}(|\alpha\rangle\langle\beta|)=-\frac{1}{2}\left(c_{J}(y, \alpha)+c_{J}(y, \beta)\right)|\alpha\rangle\langle\beta| .
$$

For $y \notin b_{x}(r), \mathcal{L}_{y}$ is not supported on $x$, and thus cannot change the configuration there. This implies that the evolution cannot change the configurations over the set $\Delta(r)$, where $\Delta=\{x \in \Lambda \mid \alpha(x) \neq \beta(x)\}$. In turn, this implies that $\mathcal{L}_{\Delta}$ commutes with $\mathcal{L}-\mathcal{L}_{\Delta}$ (since it acts as a Schur multiplier whose entries depend only on the sites in $\Delta(r))$. Finally, this means that

$$
\begin{aligned}
\left\|e^{t \mathcal{L}_{\Lambda}}(|\alpha\rangle\langle\beta|)\right\|_{1} & \leqslant\left\|e^{t \mathcal{L}_{\Delta}}(|\alpha\rangle\langle\beta|)\right\|_{1}=\exp \left(-t \frac{1}{2}\left(\sum_{x \in \Delta} c_{J}(x, \alpha)+c_{J}(x, \beta)\right)\right) \\
& \leqslant \exp \left(-t \frac{1}{2} c_{m} d(\alpha, \beta)\right) \rightarrow 0 .
\end{aligned}
$$

Since the off-diagonal elements are killed, $\rho$ must be of the form $\sum_{\sigma} p_{\sigma}|\sigma\rangle\langle\sigma|$. Writing the equation $\mathcal{L}_{\Lambda}(\rho)=0$ we obtain

$$
\sum_{\sigma} \sum_{x} c_{J}(x, \sigma) p_{\sigma}\left|\sigma^{x}\right\rangle\left\langle\sigma^{x}\left|-\sum_{\sigma} \sum_{x} c_{J}(x, \sigma) p_{\sigma}\right| \sigma\right\rangle\langle\sigma|=0
$$

which implies

$$
\sum_{x} c_{J}\left(x, \sigma^{x}\right) p_{\sigma^{x}}=\sum_{x} p_{\sigma} c_{J}(x, \sigma)
$$

The last equation is simply a rewriting of the fact that $\left(p_{\sigma}\right)$ is a stationary distribution for $Q_{\Lambda}$, that is, it is exactly a Gibbs state on $\Lambda$.

Since $\mathcal{L}_{\Lambda}$ and $\mathcal{L}_{\Lambda}+\mathcal{D}$ have the same stationary distributions, even locally, all properties that depend just on the structure of the fixed-point sets will be shared by both: this is the case, for example, of frustration freeness (which we will prove next) and LTQO (which will be proved later).

Proposition 7.8. $\mathcal{L}_{\Lambda}$ (and consequently $\mathcal{L}_{\Lambda}+\mathcal{D}$ ) is frustration free.

Proof. By the previous proposition, we have that $\mathcal{X}_{\mathcal{L}_{\Lambda}}=\mathcal{G}(\Lambda)$. We know 41] that for Gibbs states it holds that

$$
\Delta \subset \Lambda \Rightarrow \mathcal{G}(\Lambda) \subset \mathcal{G}(\Delta),
$$

but this is exactly the frustration-freeness condition for $\mathcal{L}_{\Lambda}$. 


\subsection{Stability of Glauber dynamics}

We want to show that the contraction of the semigroup generated by $\mathcal{L}_{\Lambda}+\mathcal{D}$ can be controlled by the contraction of the classical Glauber dynamics. To fix notation, denote by $\mathcal{C}: \mathcal{A}_{\Lambda} \rightarrow \mathcal{A}_{\Lambda}$ the projector on the diagonal subspace with respect to the computational basis. $\mathcal{C}$ is a completely positive, trace preserving map, and it also satisfies $\mathcal{C}=\lim _{t \rightarrow \infty} \exp (t \mathcal{D})$. Since $\mathcal{L}_{\Lambda}$ commutes with $\mathcal{D}$, it also commutes with $\mathcal{C}$. Then we can prove the following:

Lemma 7.9. If $T_{t}=\exp \left(t\left(\mathcal{L}_{\Lambda}+\mathcal{D}\right)\right)$, then

$$
\eta\left(T_{t}\right) \leqslant \eta\left(T_{t} \circ \mathcal{C}\right)+\eta(\exp (t \mathcal{D}))
$$

Proof. Fix an initial state $\rho$. Then we can write

$$
\begin{aligned}
\left\|T_{t}(\rho)-T_{\infty}(\rho)\right\|_{1} & \leqslant\left\|T_{t} \circ \mathcal{C}(\rho)-T_{\infty}(\rho)\right\|_{1}+\left\|T_{t} \circ(1-\mathcal{C})(\rho)\right\|_{1} \\
& \leqslant\left\|T_{t} \circ \mathcal{C}(\rho)-T_{\infty} \circ \mathcal{C}(\rho)\right\|_{1}+\|\exp (t \mathcal{D}) \circ(1-\mathcal{C})(\rho)\|_{1} \\
& \leqslant \eta\left(T_{t} \circ \mathcal{C}\right)+\eta(\exp (t \mathcal{D})),
\end{aligned}
$$

where we have used the fact that $\mathcal{L}_{\Lambda}$ and $\mathcal{D}$ commute, and that the fixed points of $\mathcal{L}_{\Lambda}$ are invariant under $\mathcal{C}$.

We know, because of theorem 4.2 , that

$$
\eta(\exp (t \mathcal{D})) \leqslant|\Lambda| e^{-\frac{\gamma}{2} t}
$$

and this implies the following result.

Corollary 7.10. If the classical Glauber dynamics satisfies rapid mixing, then also the quantum embedded Glauber dynamics generated by $\mathcal{L}_{\Lambda}+\mathcal{D}$ does.

Remark 7.11. Convergence rates of classical Glauber dynamics are a well studied subject. It is known that, in some regimes, classical Glauber dynamics satisfies a Log Sobolev inequality with system-size independent Log Sobolev constant (for a review on the subject see [4]). In such situations the classical chain has a logarithmic mixing time, and thus satisfies rapid mixing.

For this class of classical dynamical systems it is possible to apply our main result 4.3 In particular, we can arbitrary perturb the transition rates $c_{J}(x, \sigma)$ by some $e(x, \sigma)$, not necessary preserving detailed balance. If we denote by $\mathcal{E}$ the maximum of $|e(x, \sigma)|$, the difference between the perturbed and the original evolution of local observables can be bounded by $\mathcal{E}$ times a factor depending on the size of the support of the observables taken into account.

Theorem 7.12. Let $Q_{\Lambda}$ the generator of a classical Glauber dynamics, having a unique fixed point and satisfying a Log Sobolev inequality with constant independent of system size. Let $E$ be the generator of another classical Markov process of the form

$$
(E f)(\sigma)=\sum_{x \in \Lambda} e(x, \sigma) \nabla_{x} f(\sigma) .
$$

Suppose that $\mathcal{E}=\sup _{x, \sigma}|e(x, \sigma)|<\infty$ and that $e(x, \cdot)$ has support bounded uniformly in $x$. Denote by $T_{t}$ the evolution generated by $Q_{\Lambda}$ and by $S_{t}$ the evolution generated by $Q_{\Lambda}+E$. Then, for each function $f$ supported on $A \subset \Lambda$, it holds that

$$
\left\|T_{t}(f)-S_{t}(f)\right\|_{\infty} \leqslant c(|A|)\|f\|_{\infty} \mathcal{E},
$$

for some $c(\cdot)$ independent of system size and polynomially growing.

Remark 7.13. It is known 43,46 that the Ising model on $\mathbb{Z}^{2}$ or $(\mathbb{Z} / n \mathbb{Z})^{2}$ has a system size independent Log Sobolev constant for high temperatures (when the inverse temperature $\beta$ is lower than the critical value $\beta_{c}$ ), or at any temperature in presence of an external magnetic field. In this regime the Glauber dynamics sampling the Ising model is stable (in the sense of theorem 4.3). 


\subsection{Weak mixing and LTQO}

As a nice observation, though not necessary to prove theorem 7.12 , we want show that weak mixing, a condition on Gibbs states defined in [47, is equivalent to the LTQO condition given in section 6 The weak mixing conditions for two-dimensional systems has been shown 46 to imply $L_{2}$ convergence of the corresponding Glauber dynamics.

Definition 7.14. We say that the Gibbs measures in $\mathcal{G}(\Lambda)$ satisfy the weak mixing condition in $V \subset \Lambda$ if there exist constants $C$ and $m$ such that, for every subset $\Delta \subset V$, the following holds:

$$
\sup _{\tau, \tau^{\prime} \in \Omega_{V^{c}}}\left\|\mu_{V, \Delta}^{\tau}-\mu_{V, \Delta}^{\tau^{\prime}}\right\|_{1} \leqslant C \sum_{\substack{x \in \Delta, y \in \partial_{r}^{+} V}} e^{-m \operatorname{dist}(x, y)},
$$

where $\partial_{r}^{+} V=\left\{x \in V^{c} \mid \operatorname{dist}(x, V) \leqslant r\right\}$ and $\mu_{V, \Delta}^{\tau}=\operatorname{tr}_{V \backslash \Delta} \mu_{V}^{\tau}$.

Proposition 7.15. If $\mathcal{G}(\Lambda)$ satisfies the weak mixing condition for each $V \subset \Lambda$, then $\mathcal{L}_{\Lambda}$ (and consequently $\left.\mathcal{L}_{\Lambda}+\mathcal{D}\right)$ satisfies LTQO.

Proof. Take $A \subset \Lambda, \ell \geqslant 0$, and let $V$ be $A(\ell)$. The weak mixing condition for $V$ implies that there exist constants $C$ and $m$ such that

$$
\sup _{\tau, \tau^{\prime} \in \Omega_{V^{c}}}\left\|\mu_{V, A}^{\tau}-\mu_{V, A}^{\tau^{\prime}}\right\|_{1} \leqslant C \sum_{\substack{x \in A \\ y \in \partial_{r}^{+} V}} e^{-m \operatorname{dist}(x, y)} \leqslant C e^{-m \ell}|A|\left|\partial_{r}^{+} A(\ell)\right| .
$$

This is the LTQO condition with $\Delta_{0}(\ell)=C e^{-m \ell}|A|\left|\partial_{r}^{+} A(\ell)\right|$. The bound, proven for states of the form $\mu_{V}^{\tau}$, can be extended by convexity to all $\mathcal{G}(V)$. Let $\eta_{0}, \eta_{1} \in \mathcal{G}(V)$. By definition, $\eta_{0}$ and $\eta_{1}$ are convex combination of states of the form $\mu_{V}^{\tau}$, thus we can write

$$
\eta_{0}=\sum_{i} p_{i} \mu_{V}^{\tau_{i}}, \quad \eta_{1}=\sum_{j} q_{j} \mu_{V}^{\sigma_{j}}, \quad \sum_{i} p_{i}=\sum_{j} q_{j}=1 ; \quad p_{i}, q_{j} \geqslant 0 .
$$

Then we have

$$
\begin{aligned}
\left\|\eta_{0, A}-\eta_{1, A}\right\|_{1} & =\left\|\sum_{i} p_{i} \mu_{V, A}^{\tau_{i}}-\sum_{j} q_{j} \mu_{V, A}^{\sigma_{j}}\right\|_{1} \\
& =\left\|\sum_{i} p_{i}\left(\sum_{j} q_{j} \mu_{V, A}^{\tau_{i}}\right)-\sum_{j} q_{j}\left(\sum_{i} p_{i} \mu_{V, A}^{\sigma_{j}}\right)\right\|_{1} \\
& \leqslant \sum_{i, j} p_{i} q_{j}\left\|\mu_{V, A}^{\tau_{i}}-\mu_{V, A}^{\sigma_{j}}\right\|_{1} \leqslant \sup _{\tau, \sigma}\left\|\mu_{V, A}^{\tau}-\mu_{V, A}^{\sigma}\right\|_{1} .
\end{aligned}
$$

\section{Conclusions and open questions}

In the context of local perturbations of local Hamiltonians, changes in the ground state can be detected by the lack of smoothness of the expectation value of local observables. Via the quasi-adiabatic technique 24], the regularity of such expectation values can be related to the study of the effect that the perturbation has on the spectral gap of the Hamiltonian. In [49], the stability of the spectral gap was shown under the assumptions of frustration-freeness and local indistinguishability between ground states of local patches of the original Hamiltonian. 
In this paper we have studied a class of open quantum systems described by local Lindbladian evolutions with unique fixed points, focusing on the problem of the smoothness of evolution of local observables in the presence of local perturbations. Given any initial configuration, the system will converge toward the fixed point with a certain rate. The slowest rate over all possible initial configurations defines a mixing property of the Lindbladian, and we consider how this scales with the system size. In the case of power-law decay of interactions, we show that a logarithmic scaling is sufficient for the stability of the evolution of local observables, while for exponentially decaying and finite range interactions a scaling at least as fast as a certain polynomial, determined by equation (41), is also sufficient. Moreover, the same assumptions imply certain properties of the fixed point, such as local topological quantum order. It should be emphasized that Log Sobolev inequalities provide strong enough convergence-time estimates to satisfy our assumptions, but that our results also apply more generally.

The most important open question involves state engineering of degenerate topologically ordered states, such as topologically protected quantum codes. For such states, all known preparation maps have a convergence time that is slower than required for our result to apply [36]. It is an interesting question whether it is possible to exploit the very weak requirements in terms of locality of the boundary condition in our definition of uniform families (see definition 3.3 to construct faster mixing maps for which one could prove stability, since logical observables partially supported on such boundaries are not necessarily localizable in the sense of 36 .

Acknowledgments T.S.C. is supported by a Royal Society University Research fellowship, and was previously supported by a Juan de la Cierva fellowship. T.S.C., A.L., and D.P.-G. are supported by Spanish grants MTM2011-26912 and QUITEMAD, and European CHIST-ERA project CQC (funded partially by MINECO grant PRI-PIMCHI-2011-1071). A.L. is supported by Spanish Ministerio de Economía y Competividad FPI fellowship BES-2012-052404. SM acknowledges funding provided by the Institute for Quantum Information and Matter, an NSF Physics Frontiers Center with support of the Gordon and Betty Moore Foundation through Grant \#GBMF1250 and by the AFOSR Grant \#FA8750-12-2-0308. The authors would like to thank the hospitality of the Centro de Ciencias Pedro Pascual in Benasque, where part of this work was carried out.

\section{Appendix A The non-stable example}

The following example will satisfy all the conditions of theorem 4.3 except forming an uniform family, and will be shown to be unstable. Interestingly, the system is rapid mixing, showing that without the correct structure with respect to system size scaling, rapid mixing alone is not sufficient to imply stability of local observables. This example is the generalization to dissipative systems of the globally gapped but not locally gapped example in [49]. We will show that the characteristics of the dynamics are essentially determined by a classical Markov chain embedded into the Lindbladian. For a general review on convergence of Markov chains, see 40 .

Example A.1. Consider a chain of $2 N$ classical spins, with values in $\{0,1\}$. Let us define a generator $Q^{2 N}$ of a classical Markov chain over the configuration space $\{0,1\}^{2 N}$. We will define $Q^{2 N}$ in a translationally- 
invariant way as follows:

$$
\begin{aligned}
& |10\rangle \quad|00\rangle \quad|11\rangle \quad|01\rangle \\
& Q_{c}=\begin{array}{l}
|10\rangle \\
|00\rangle \\
|11\rangle \\
|01\rangle
\end{array}\left(\begin{array}{cccc}
-\frac{2}{3 N} & 0 & 0 & \frac{2}{3 N} \\
0 & -1 & 0 & 1 \\
0 & 0 & -1 & 1 \\
0 & 0 & 0 & 0
\end{array}\right), \quad Q_{r}=\begin{array}{l}
|10\rangle \\
|00\rangle \\
|11\rangle
\end{array}\left(\begin{array}{cccc}
-1 & 0 & 1 & 0 \\
0 & -1 & 0 & 1 \\
0 & 0 & 0 & 0 \\
0 & 0 & 0 & 0
\end{array}\right), \\
& |10\rangle \quad|00\rangle \quad|11\rangle \quad|01\rangle \\
& Q_{l}=\begin{array}{l}
|10\rangle \\
|00\rangle \\
|11\rangle \\
|01\rangle
\end{array}\left(\begin{array}{cccc}
-1 & 1 & 0 & 0 \\
0 & 0 & 0 & 0 \\
0 & 0 & -1 & 1 \\
0 & 0 & 0 & 0
\end{array}\right), \quad \delta_{0}=|0\rangle\left\langle 0\left|, \quad \delta_{1}=\right| 1\right\rangle\langle 1| .
\end{aligned}
$$

We then define for each $i=1 \ldots N$, a generator matrix $Q_{i}$ acting on spins $(2 i-2, \ldots, 2 i+1)$ by

$$
Q_{i}=\mathbb{1} \otimes Q_{c} \otimes \mathbb{1}+\mathbb{1} \otimes Q_{r} \otimes \delta_{0}+\delta_{1} \otimes Q_{l} \otimes \mathbb{1}
$$

and $Q^{2 N}=\sum_{i=1}^{N} Q_{i}$.

The matrix $Q_{i}$ can only change spins $(2 i-1,2 i)$ : its transition graph restricted to such spins is presented in figure 3

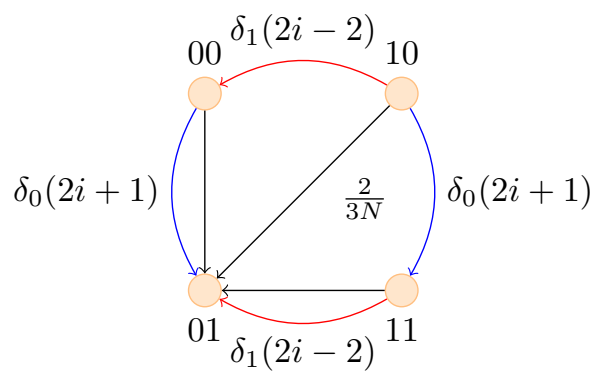

$|10\rangle$
$|00\rangle$
$|11\rangle$
$|01\rangle$$\left(\begin{array}{cccc}* & |00\rangle & |11\rangle & |01\rangle \\ 0 & * & 1 & \frac{2}{3 N} \\ 0 & 0 & * & 1+1 \\ 0 & 0 & 0 & 0\end{array}\right)$

Figure 3: The transition matrix for $Q_{i}$ on the spins $(2 i-1,2 i)$. The blue and the red transitions are present depending on the nearby sites: the blue ones if there is a 0 on the right, the red ones if there is a 1 on the left. Asterisks in the diagonal are such that the sum of each row is zero.

By construction, $Q^{2 N}$ is upper triangular. Thus the elements on the diagonal are the eigenvalues. The unique steady state is then $|0101 \ldots 01\rangle$, and the smallest non-zero eigenvalue, corresponding to the state $|1010 \ldots 10\rangle$, is $\frac{2}{3}$. Furthermore, it is easy to see that the diameter of the graph of the transitions of $Q^{2 N}$ is $N$, and in turn this implies that the mixing time for $Q^{2 N}$ is of order $O(\log N)^{10}$

Let us now embed this classical Markov chain into a Lindbad operator, in a similar fashion as we have done in section 7 with Glauber dynamics. We will consider then a chain of $2 N$ qubits, and define the following Lindblad operators: if $k$ is odd, then

$$
\begin{aligned}
L_{k, 1} & =\sigma_{x}^{k+1}|0\rangle\left\langle\left. 0\right|_{k} \otimes \mid 0\right\rangle\left\langle\left. 0\right|_{k+1},\right. \\
L_{k, 2} & =\sigma_{x}^{k} \quad|1\rangle\left\langle\left. 1\right|_{k} \otimes \mid 1\right\rangle\left\langle\left. 1\right|_{k+1},\right. \\
L_{k, 3} & =\sqrt{\frac{2}{3 N}} \sigma_{x}^{k} \otimes \sigma_{x}^{k+1}|1\rangle\left\langle\left. 1\right|_{k} \otimes \mid 0\right\rangle\left\langle\left. 0\right|_{k+1} ;\right.
\end{aligned}
$$

\footnotetext{
10 This can be seen from the upper triangular form of $Q^{2 N}$, noticing that the polynomials appearing in $e^{t Q^{2 N}}$ have degree of at most the diameter of the transition graph.
} 
if $k$ is even, then

$$
\begin{aligned}
& L_{k, 1}=\sigma_{x}^{k} \quad|0\rangle\left\langle\left. 0\right|_{k} \otimes \mid 0\right\rangle\left\langle\left. 0\right|_{k+1},\right. \\
& L_{k, 2}=\sigma_{x}^{k+1}|1\rangle\left\langle\left. 1\right|_{k} \otimes \mid 1\right\rangle\left\langle\left. 1\right|_{k+1},\right. \\
& L_{k, 3}=0 .
\end{aligned}
$$

The Lindbladian is then defined translationally-invariantly as

$$
\mathcal{L}^{2 N}=\sum_{k=1}^{2 N} \sum_{i=1}^{3} \mathcal{L}_{k, i}+\mathcal{D}_{k} ;
$$

where $\mathcal{D}_{k}$ is a dephasing channel acting on site $k$, as in equation (44). Since $L_{k, 3}$ depends on $N$, the family we have defined is not a uniform family.

It is easy to see that the action of $\mathcal{L}^{2 N}$ on diagonal states of the form $|\alpha\rangle\langle\alpha|$, with $\alpha \in\{0,1\}^{2 N}$, is equal to that of $Q^{2 N}$ acting on $\alpha$ : this is indeed an embedding of $Q^{2 N}$.

Then, by a similar argument as in section 7, we can prove that the fixed points of $\mathcal{L}^{2 N}$ are exactly the same as those of $Q^{2 N}$ (namely, the unique state $|0101 \ldots 01\rangle\langle 0101 \ldots 01|)$, and that the mixing time of $\mathcal{L}^{2 N}$ is bounded by the sum of the mixing times of $Q^{2 N}$ and of $\mathcal{D}$. Since both of them are mixing in time $O(\log N)$, we see that $\mathcal{L}^{2 N}$ satisfies rapid mixing.

But the system is unstable: if we perturb $\mathcal{L}^{2 N}$ by removing the terms generated by $L_{k, 3}$ (which is a perturbation of order $\left.O\left(\frac{1}{N}\right)\right)$, the diagonal state $|1010 \ldots 10\rangle\langle 1010 \ldots 10|$ becomes a stationary state, and it is clearly locally ortogonal from the original one $|0101 \ldots 01\rangle\langle 0101 \ldots 01|$.

\section{References}

[1] R. Alicki et al. "On Thermal Stability of Topological Qubit in Kitaev's 4D Model". In: Open Systems 83 Information Dynamics 17.01 (2010), pp. 1-20. arXiv:0811.0033 [quant-ph]

[2] H. Araki and G. L. Sewell. "KMS conditions and local thermodynamical stability of quantum lattice systems". In: Communications in Mathematical Physics 52.2 (1977), pp. 103-109.

[3] A. Aspuru-Guzik and P. Walther. "Photonic quantum simulators". In: Nature Physics 8.4 (2012), pp. $285-291$.

[4] R. Augusiak et al. "Quantum kinetic Ising models". In: New Journal of Physics 12.2, 025021 (Feb. 2010), p. 025021. arXiv:0911.0624 [quant-ph]

[5] J. T. Barreiro et al. "Experimental multiparticle entanglement dynamics induced by decoherence". In: Nature Physics 6 (Dec. 2010), pp. 943-946. arXiv:1005.1965 [quant-ph]

[6] T. Barthel and M. Kliesch. "Quasilocality and Efficient Simulation of Markovian Quantum Dynamics". In: Physical review letters 108.23 (2012), p. 230504.

[7] R. Blatt and C. Roos. "Quantum simulations with trapped ions". In: Nature Physics 8.4 (2012), pp. 277-284.

[8] I. Bloch, J. Dalibard, and S. Nascimbène. "Quantum simulations with ultracold quantum gases". In: Nature Physics 8.4 (2012), pp. 267-276.

[9] T Bodineau and B Zegarlinski. "Hypercontractivity via spectral theory". In: Infinite Dimensional Analysis, Quantum Probability and Related Topics 3.01 (2000), pp. 15-31.

[10] S. Bravyi, M. B. Hastings, and S. Michalakis. "Topological quantum order: Stability under local perturbations". In: Journal of Mathematical Physics 51.9 (Sept. 2010), p. 093512. arXiv 1001.0344 [quant-ph].

[11] H. Briegel et al. "Measurement-based quantum computation". In: Nature Physics 5.1 (2009), pp. 19 26. 
[12] E. Dennis et al. "Topological quantum memory". In: Journal of Mathematical Physics 43 (Sept. 2002), pp. 4452-4505. eprint: arXiv:quant-ph/0110143.

[13] L.-M. Duan and C. Monroe. "Colloquium: Quantum networks with trap-ped ions". In: Rev. Mod. Phys. 82 (2 Apr. 2010), pp. 1209-1224.

[14] E. Farhi et al. "A Quantum Adiabatic Evolution Algorithm Applied to Random Instances of an NPComplete Problem". In: Science 292.5516 (2001), pp. 472-475.

[15] V. Gorini, A. Kossakowski, and E. C. G. Sudarshan. "Completely positive dynamical semigroups of N-level systems". In: J. Mathematical Phys. 17.5 (1976), pp. 821-825. Issn: 0022-2488.

[16] L. Gross. "Hypercontractivity, logarithmic Sobolev inequalities, and applications: a survey of surveys". In: Diffusion, quantum theory, and radically elementary mathematics. Vol. 47. Math. Notes. Princeton, NJ: Princeton Univ. Press, 2006, pp. 45-73.

[17] L. Gross. "Logarithmic Sobolev inequalities". In: Amer. J. Math. 97.4 (1975), pp. 1061-1083. ISSN: 0002-9327.

[18] L. Gross. "Logarithmic Sobolev inequalities and contractivity properties of semigroups". In: Dirichlet forms (Varenna, 1992). Vol. 1563. Lecture Notes in Math. Berlin: Springer, 1993, pp. 54-88.

[19] J. Haah. "Local stabilizer codes in three dimensions without string logical operators". In: Phys. Rev. A 83.4, 042330 (Apr. 2011), p. 042330. arXiv:1101.1962 [quant-ph]

[20] K. Hammerer, A. S. Sørensen, and E. S. Polzik. "Quantum interface between light and atomic ensembles". In: Rev. Mod. Phys. 82 (2 Apr. 2010), pp. 1041-1093.

[21] M. B. Hastings. "An area law for one-dimensional quantum systems". In: Journal of Statistical Mechanics: Theory and Experiment 2007.08 (2007), P08024. arXiv:0705.2024 [quant-ph]

[22] M. B. Hastings. "Lieb-Schultz-Mattis in higher dimensions". In: Phys. Rev. B 69 (10 Mar. 2004), p. 104431. arXiv:cond-mat/0305505.

[23] M. B. Hastings. "Locality in Quantum Systems". In: ArXiv e-prints (Aug. 2010). arXiv:1008.5137 [math-ph].

[24] M. B. Hastings and X.-G. Wen. "Quasiadiabatic continuation of quantum states: The stability of topological ground-state degeneracy and emergent gauge invariance". In: Phys. Rev. B 72 (4 July 2005), p. 045141.

[25] M. B. Hastings and T. Koma. "Spectral Gap and Exponential Decay of Correlations". English. In: Communications in Mathematical Physics 265 (3 2006), pp. 781-804. ISSN: 0010-3616. arXiv math$\mathrm{ph} / 0507008$

[26] M. Hein et al. "Entanglement in graph states and its applications". In: Quantum computers, algorithms and chaos. Vol. 162. Proc. Internat. School Phys. Enrico Fermi. IOS, Amsterdam, 2006, pp. 115-218.

[27] R. Holley. "Possible rates of convergence in finite range, attractive spin systems". In: Particle systems, random media and large deviations (Brunswick, Maine, 1984). Vol. 41. Contemp. Math. Providence, RI: Amer. Math. Soc., 1985, pp. 215-234.

[28] A. A. Houck, H. E. Türeci, and J. Koch. "On-chip quantum simulation with superconducting circuits". In: Nature Physics 8.4 (2012), pp. 292-299.

[29] N. Johnston, D. W. Kribs, and V. I. Paulsen. "Computing stabilized norms for quantum operations via the theory of completely bounded maps". In: Quantum Inf. Comput. 9.1-2 (2009), pp. 16-35. ISSN: 1533-7146. arXiv:0711.3636 [quant-ph].

[30] S. P. Jordan, K. S. Lee, and J. Preskill. "Quantum algorithms for quantum field theories". In: Science 336.6085 (2012), pp. 1130-1133.

[31] M. J. Kastoryano and K. Temme. "Quantum logarithmic Sobolev inequalities and rapid mixing". In: Journal of Mathematical Physics 54.5 (May 2013), p. 052202. arXiv 1207.3261 [quant-ph]. 
[32] M. J. Kastoryano, D. Reeb, and M. M. Wolf. "A cutoff phenomenon for quantum Markov chains". In: J. Phys. A 45.7 (2012), pp. 075307, 16. ISSN: 1751-8113. arXiv:1111.2123 [quant-ph]

[33] C. King. "Hypercontractivity for Semigroups of Unital Qubit Channels". In: Comm. Math. Phys. 328.1 (2014), pp. 285-301. ISSN: 0010-3616.

[34] A. Kitaev. "Fault-tolerant quantum computation by anyons". In: Annals of Physics 303.1 (2003), pp. 2 -30. ISSN: 0003-4916. arXiv:quant-ph/9707021.

[35] I. Klich. "On the stability of topological phases on a lattice". In: Annals of Physics 325 (2010), p. 2120.

[36] R. König and F. Pastawski. "Generating topological order: No speedup by dissipation". In: Phys. Rev. B 90 (4 2014), p. 045101. arXiv:1310.1037 [quant-ph].

[37] A. Kossakowski et al. "Quantum detailed balance and KMS condition". In: Comm. Math. Phys. 57.2 (1977), pp. 97-110. ISSN: 0010-3616.

[38] B. Kraus et al. "Preparation of entangled states by quantum Markov processes". In: Phys. Rev. A 78.4, 042307 (Oct. 2008). arXiv:0803.1463 [quant-ph].

[39] H. Krauter et al. "Entanglement Generated by Dissipation and Steady State Entanglement of Two Macroscopic Objects". In: Phys. Rev. Lett. 107 (8 Aug. 2011), p. 080503.

[40] D. A. Levin, Y. Peres, and E. L. Wilmer. Markov chains and mixing times. With a chapter by James G. Propp and David B. Wilson. Providence, RI: American Mathematical Society, 2009, pp. xviii+371. ISBN: 978-0-8218-4739-8.

[41] T. M. Liggett. Interacting particle systems. Classics in Mathematics. Re-print of the 1985 original. Berlin: Springer-Verlag, 2005, pp. xvi+496. ISBN: 3-540-22617-6.

[42] G. Lindblad. "On the generators of quantum dynamical semigroups". In: Comm. Math. Phys. 48.2 (1976), pp. 119-130. ISSN: 0010-3616.

[43] E. Lubetzky and A. Sly. "Cutoff for the Ising model on the lattice". In: Invent. Math. 191.3 (2013), pp. 719-755. ISSN: 0020-9910. arXiv:0909.4320 [math.PR]

[44] W. A. Majewski. "The detailed balance condition in quantum statistical mechanics". In: J. Math. Phys. 25.3 (1984), pp. 614-616. ISSN: 0022-2488.

[45] W. A. Majewski and R. F. Streater. "Detailed balance and quantum dynamical maps". In: J. Phys. A 31.39 (1998), pp. 7981-7995. ISSN: 0305-4470.

[46] F. Martinelli, E. Olivieri, and R. H. Schonmann. "For 2-D lattice spin systems weak mixing implies strong mixing". In: Comm. Math. Phys. 165.1 (1994), pp. 33-47. ISSN: 0010-3616.

[47] F. Martinelli. "Lectures on Glauber dynamics for discrete spin models". In: Lectures on probability theory and statistics (Saint-Flour, 1997). Vol. 1717. Lecture Notes in Math. Berlin: Springer, 1999, pp. 93-191.

[48] P. C. Maurer et al. "Room-Temperature Quantum Bit Memory Exceeding One Second". In: Science 336.6086 (2012), pp. 1283-1286.

[49] S. Michalakis and J. P. Zwolak. "Stability of Frustration-Free Hamiltonians". In: Communications in Mathematical Physics 322 (Sept. 2013), pp. 277-302. arXiv:1109.1588 [quant-ph]

[50] B. Nachtergaele, A. Vershynina, and V. A. Zagrebnov. "Lieb-Robinson bounds and existence of the thermodynamic limit for a class of irreversible quantum dynamics". In: Entropy and the quantum II. Vol. 552. Contemp. Math. Providence, RI: Amer. Math. Soc., 2011, pp. 161-175. arXiv:1103.1122 [math-ph].

[51] C. Nayak et al. "Non-Abelian anyons and topological quantum computation". In: Rev. Mod. Phys. 80 (3 Sept. 2008), pp. 1083-1159. arXiv:0707.1889 [cond-mat.str-el].

[52] R. Olkiewicz and B. Zegarlinski. "Hypercontractivity in Noncommutative $L_{p}$ Spaces". In: Journal of functional analysis 161.1 (1999), pp. 246-285. 
[53] F. Pastawski, L. Clemente, and J. I. Cirac. "Quantum memories based on engineered dissipation". In: Physical Review A 83.1 (2011), p. 012304.

[54] D. Poulin. "Lieb-Robinson Bound and Locality for General Markovian Quantum Dynamics". In: Phys. Rev. Lett. 104.19, 190401 (May 2010), p. 190401. arXiv 1003.3675 [quant-ph].

[55] S. Sachdev. Quantum Phase Transitions. John Wiley \& Sons, Ltd, 2007. ISBN: 9780470022184.

[56] G. L. Sewell. "KMS conditions and local thermodynamical stability of quantum lattice systems. II". In: Communications in Mathematical Physics 55.1 (1977), pp. 53-61.

[57] O. Szehr and M. M. Wolf. "Perturbation bounds for quantum Markov processes and their fixed points". In: Journal of Mathematical Physics 54.3 (Mar. 2013), p. 032203. arXiv:1210.1171 [math-ph]

[58] O. Szehr, D. Reeb, and M. M. Wolf. "Spectral Convergence Bounds for Classical and Quantum Markov Processes". English. In: Communications in Mathematical Physics (2014), pp. 1-31. ISSN: 0010-3616. arXiv 1301.4827 [quant-ph].

[59] K. Temme, F. Pastawski, and M. J. Kastoryano. "Hypercontractivity of quasi-free quantum semigroups". In: ArXiv e-prints (Mar. 2014). arXiv:1403.5224 [quant-ph]

[60] K. Temme et al. "The $\chi^{2}$-divergence and mixing times of quantum Markov processes". In: Journal of Mathematical Physics 51.12, 122201 (2010), p. 122201. arXiv:1005.2358 [quant-ph]

[61] F. Verstraete, M. M. Wolf, and J. I. Cirac. "Quantum computation and quantum-state engineering driven by dissipation". In: Nature. Physics 5.9 (2009), pp. 633-636. arXiv:0803.1447 [quant-ph]

[62] M. M. Wolf and D. Perez-Garcia. "The inverse eigenvalue problem for quantum channels". In: ArXiv e-prints (May 2010). arXiv:1005.4545 [quant-ph]

[63] M. M. Wolf. Quantum Channels \&3 Operations. Guided Tour. 2012. eprint: http://www-m5.ma.tum. de/foswiki/pub/M5/Allgemeines/MichaelWolf/QChannelLecture.pdf. 